\title{
Proteolytic Processing of Neurexins by Presenilins Sustains Synaptic Vesicle Release
}

\author{
Emilia Servián-Morilla, ${ }^{1,2}$ ¿Estefanía Robles-Lanuza, ${ }^{1,2 *}{ }^{\oplus}$ Ana C. Sánchez-Hidalgo, ${ }^{1,2 *}$ Rafael J. Camacho-Garcia, ${ }^{1,2}$

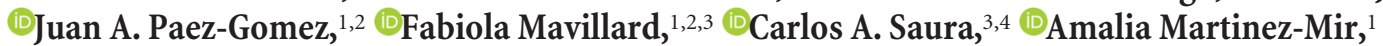 \\ and $\odot$ Francisco G. Scholl 1,2 \\ ${ }^{1}$ Instituto de Biomedicina de Sevilla (IBiS), Hospital Universitario Virgen del Rocío/Consejo Superior de Investigaciones Científicas (CSIC)/Universidad de \\ Sevilla, 41013 Sevilla, Spain, ${ }^{2}$ Departamento de Fisiología Médica y Biofísica, Facultad de Medicina, Universidad de Sevilla, 41009 Sevilla, Spain, ${ }^{3}$ Centro de \\ Investigación Biomédica en Red sobre Enfermedades Neurodegenerativas, 28031 Madrid, Spain, and ${ }^{4}$ Institut de Neurociències, Departament de \\ Bioquímica i Biologia Molecular, Universitat Autònoma de Barcelona, 08193 Barcelona, Spain
}

Proteolytic processing of synaptic adhesion components can accommodate the function of synapses to activity-dependent changes. The adhesion system formed by neurexins (Nrxns) and neuroligins (Nlgns) bidirectionally orchestrate the function of presynaptic and postsynaptic terminals. Previous studies have shown that presenilins (PS), components of the gamma-secretase complex frequently mutated in familial Alzheimer's disease, clear from glutamatergic terminals the accumulation of Nrxn C-terminal fragments (Nrxn-CTF) generated by ectodomain shedding. Here, we characterized the synaptic consequences of the proteolytic processing of Nrxns in cultured hippocampal neurons from mice and rats of both sexes. We show that activation of presynaptic Nrxns with postsynaptic Nlgn1 or inhibition of ectodomain shedding in axonal Nrxn $1-\beta$ increases presynaptic release at individual terminals, likely reflecting an increase in the number of functional release sites. Importantly, inactivation of PS inhibits presynaptic release downstream of Nrxn activation, leaving synaptic vesicle recruitment unaltered. Glutamate-receptor signaling initiates the activity-dependent generation of Nrxn-CTF, which accumulate at presynaptic terminals lacking PS function. The sole expression of Nrxn-CTF decreases presynaptic release and calcium flux, recapitulating the deficits due to loss of PS function. Our data indicate that inhibition of Nrxn processing by PS is deleterious to glutamatergic function.

Key words: Alzheimer's disease; neurexins; neuroligins; presenilins; synapse; synaptic adhesion

Significance Statement

To gain insight into the role of presenilins (PS) in excitatory synaptic function, we address the relevance of the proteolytic processing of presynaptic neurexins (Nrxns) in glutamatergic differentiation. Using synaptic fluorescence probes in cultured hippocampal neurons, we report that trans-synaptic activation of Nrxns produces a robust increase in presynaptic calcium levels and neurotransmitter release at individual glutamatergic terminals by a mechanism that depends on normal PS activity. Abnormal accumulation of Nrxn C-terminal fragments resulting from impaired PS activity inhibits presynaptic calcium signal and neurotransmitter release, assigning synaptic defects to Nrxns as a specific PS substrate. These data may provide links into how loss of PS activity inhibits glutamatergic synaptic function in Alzheimer's disease patients.

\section{Introduction}

Interaction between synaptic adhesion proteins mediates the formation and function of synaptic contacts as they organize supra-

\footnotetext{
Received May 18, 2017; revised Nov. 3, 2017; accepted Nov. 26, 2017.

Author contributions: E.S.-M., E.R.-L., and F.G.S. designed research; E.S.-M., E.R.-L., A.C.S.-H., and R.J.C.-G. performed research; F.M. and C.A.S. contributed unpublished reagents/analytic tools; E.S.-M., E.R.-L., A.C.S.-H., J.A.P.-G., A.M.-M., and F.G.S. analyzed data; A.M.-M. and F.G.S. wrote the paper.

This work was funded by Junta de Andalucía (Grant P11-CVI-7599) and Ministerio de Economía y Competitividad (MINECO) (Grant BFU2015-71464-R) and cofunded by Fondo Europeo de Desarrollo Regional (FEDER) funds. E.S.-M. was a recipient of a fellowship from Junta de Andalucía. E.R.-L. is a fellow from V Plan Propio de Investigación (Universidad de Sevilla). A.C.S.-H. received a fellowship from Junta de Andalucía. Part of the study was performed at
}

molecular complexes at both sides of the synapse. Proteolytic processing of synaptic proteins is a posttranslational mechanism

\footnotetext{
Centro de Investigación Tecnológica e Innovación (CITIUS) (Universidad de Sevilla). We thank J. Shen for providing fPS1/fPS1 and PS2 ${ }^{-1-}$ mice and Cre constructs, D. Selkoe for PS1 vectors, L. Lagnado for the gift of the SypHy construct, F.J. Morón from the IBiS Genomics Service for technical support and Rafael Fernández-Chacón for discussion and critical reading of the manuscript.

The authors declare no competing financial interests.

${ }^{*}$ E.R.-L. and A.C.S.-H. contributed equally to this work

Correspondence should be addressed to Francisco G. Scholl, Instituto de Biomedicina de Sevilla (IBiS), Avda Manuel Siurot s/n, 41013 Sevilla, Spain. E-mail: fgs@us.es.

DOI:10.1523/JNEUROSCI.1357-17.2017

Copyright $\odot 2018$ the authors $\quad 0270-6474 / 18 / 380901-17 \$ 15.00 / 0$
} 
that regulates the function of synapses upon activity-dependent changes, whereas impairment of normal proteolytic processing often results in synaptic dysfunction (Saura et al., 2004; Nagy et al., 2006; Huntley, 2012; Prox et al., 2013). Neurexins (Nrxn1, Nrxn2, and Nrxn3) are presynaptic membrane proteins that link neurotransmitter release with adhesion to postsynaptic partners. The use of different promoters and alternative splicing at the extracellular domain result in >1000 Nrxn isoforms (Tabuchi and Südhof, 2002; Schreiner et al., 2014; Fuccillo et al., 2015; Schreiner et al., 2015). The highly polymorphic extracellular domain of Nrxns engages in trans-synaptic interactions with postsynaptic proteins, whereas the conserved cytoplasmic domain recruits synaptic ligands and regulates neurotransmitter release (Boucard et al., 2005; Chih et al., 2006; Ko et al., 2009; Rabaneda et al., 2014; Aoto et al., 2015). Neuroligins (Nlgn1, Nlgn2, Nlgn3, Nlgn4X, and Nlgn4Y) are postsynaptic partners of Nrxns that regulate several aspects of glutamatergic and GABAergic differentiation (Ichtchenko et al., 1995; Baudouin and Scheiffele, 2010). At glutamatergic synapses, Nlgn1 increases synapse density and stimulates neurotransmitter release probability by a mechanism that depends on trans-synaptic interaction with Nrxns (Dean et al., 2003; Chih et al., 2006; Futai et al., 2007). Mutations in NLGN3, NLGN4X, and NRXN1 genes have been identified in neurodevelopmental disorders including autism and schizophrenia (Jamain et al., 2003; Laumonnier et al., 2004; Kim et al., 2008; Rujescu et al., 2009; Camacho-Garcia et al., 2012; Camacho-Garcia et al., 2013; Lowther et al., 2017), suggesting a causative role for synaptic dysfunction of Nrxns and Nlgns in brain diseases.

Apart from their role in neurodevelopmental disorders, recent findings suggest a malfunctioning of the Nrxn-Nlgn 1 glutamatergic pathway in age-associated diseases such as Alzheimer's disease (AD) (Martinez-Mir et al., 2013; Bie et al., 2014; Sindi et al., 2014; Tristán-Clavijo et al., 2015). We and others have shown that presenilins (PS1 and PS2), the catalytic subunit of the gammasecretase complex mutated in familial $\mathrm{AD}$ (FAD), cleave the C-terminal fragment (CTF) of Nlgn1 and Nrxn isoforms (Bot et al., 2011; Saura et al., 2011; Peixoto et al., 2012; Suzuki et al., 2012). Nrxn-CTF accumulate at glutamatergic terminals of cultured hippocampal neurons defective in PS/gamma-secretase activity and at presynaptic fractions of PS1 and PS2 conditional double knock-out (PS cDKO) mice that lack PS expression in forebrain glutamatergic neurons (Saura et al., 2004; Saura et al., 2011). FAD mutations in PS1 are thought to act by a partial loss-of-function mechanism (Shen and Kelleher, 2007; Xia et al., 2015; Sun et al., 2017). Interestingly, several FAD-linked PS1 mutants fail to clear Nrxn-CTF (Bot et al., 2011; Saura et al., 2011). Therefore, based on the accumulation of Nrxn-CTF by loss of PS function, an unexplored hypothesis is that failure of the proteolytic processing of Nrxns mediates synaptic defects at glutamatergic terminals lacking PS activity.

In this study, we show that proteolytic processing of Nrxns by PS is required to maintain synaptic vesicle release at glutamatergic terminals. Using fluorescent synaptic probes, we found that Nlgn 1 increases presynaptic release and evoked calcium levels at contacting terminals. Genetic inactivation of PS or inhibition of PS/gamma-secretase activity decreases synaptic vesicle release and presynaptic calcium levels at glutamatergic terminals induced by the Nrxn-Nlgn 1 complex. However, synapse formation and recruitment of synaptic vesicles induced by Nlgn1 were not affected by inhibition of PS function. We found that the production of Nrxn-CTF is initiated by an activity-dependent mechanism that requires NMDA/AMPA receptor function. By mutational screening, we identified a conserved region in the juxtamembrane domain of Nrxn1 responsible for the generation of Nrxn-CTF. Importantly, the sole expression of exogenous Nrxn-CTF at presynaptic terminals decreased evoked presynaptic release and activitydependent calcium levels in neurons with unaltered PS function, thereby mimicking the effect of the loss of PS function at glutamatergic synapses.

\section{Materials and Methods}

DNA and lentiviral vectors. For expression experiments, pCAG-GS and lentiviral vectors encoding DsRed, HA-Nrxn1- $\beta$, HA-neuroligin $1(A B)$, SypHy, hPS1, and PS1 D385A were described previously (Taniguchi et al., 2007; Saura et al., 2011; Rabaneda et al., 2014). Mutants harboring deletions in the juxtamembrane domain of human Nrxn1 were generated by PCR with specific forward primers containing a SacI site and a common reverse primer containing a BglII site at the $5^{\prime}$ ends. PCR products were digested with SacI-BglII and cloned into human HA-Nrxn1- $\beta$ (Camacho-Garcia et al., 2012). For the generation of HA-Nrxn-CTF, a PCR fragment coding for the $85 \mathrm{C}$-terminal residues of human Nrnx-1 was inserted in-frame downstream of a Nlgn 1 signal peptide, followed by an HA tag, and cloned into pCAG-GS. An HA-Nrxn-CTF $\triangle$ PDZ construct lacking the three C-terminal residues of human Nrxn1 was generated by PCR with specific primers. The lentiviral vectors driving Cre and $\Delta$ Cre expression under the ubiquitin C promoter were shown previously to inactivate PS1 efficiently in $\mathrm{fPS} 1 / \mathrm{fPS} 1 ; \mathrm{PS} 2^{-/-}$primary neurons $(\mathrm{Ho}$ et al., 2006; Watanabe et al., 2009). For the generation of the SyGCaMP3 construct, the DNA fragment coding for GCaMP2 in SyGCaMP2 vector (catalog \#26124; Addgene) (Dreosti et al., 2009) was exchanged by a fragment coding for GCaMP3 (catalog \#22692; Addgene) (Tian et al., 2009). For lentiviral expression, HA-Nrxn-CTF, HA-Nrxn-CTF $\Delta$ PDZ, and SyGCaMP3 constructs were inserted in a lentiviral vector containing the human synapsin promoter (Gascón et al., 2008). Generation of recombinant lentiviral particles and infection of hippocampal cultures with concentrated lentivirus were performed as described previously (Gascón et al., 2008).

Cell culture, transfection, and immunofluorescence. Hippocampal cultures were obtained from embryonic day 18 (E18) to E19 rat brains or from E18-E19 fPS1/fPS1; PS2 ${ }^{-1-}$ mouse embryos of either sex (RRID: MGI:3777984, RRID:MGI:2664243). Dissociated cells were plated at $35.000 \mathrm{cells} / \mathrm{cm}^{2}$ on poly-D-lysine (Sigma-Aldrich)-coated glass coverslips and maintained in neurobasal medium (Invitrogen) supplemented with B27 (Thermo Fisher Scientific), Glutamax, and penicillin/streptomycin (Invitrogen). HEK293T cultures (RRID:CVCL_0063) were grown in DMEM containing 10\% fetal bovine serum (HyClone), Glutamax, and penicillin/streptomycin. Cultured hippocampal neurons and HEK293T cells were transfected with Lipofectamine 2000 (Invitrogen) following the manufacturer's recommendations. For immunofluorescence experiments, cultures were fixed with 4\% PFA and permeabilized with $0.05 \%$ Triton X-100 (Sigma-Aldrich). The following primary antibodies were used: rat anti-HA (1:1000; clone 3F10; Roche; RRID:AB_390918), mouse anti-CASK (1:200; BD Transduction Lab; RRID:AB_398103) and rabbit anti-VGluT1 (1:1500; Synaptic Systems; RRID:AB_887877). A chicken anti cyto-Nrxn antibody has been described previously (Dean et al., 2003). Preabsorbed donkey antibodies conjugated with Cy2, Cy3, and Cy5 fluorophores were used as secondary antibodies (1:800; Jackson ImmunoResearch; RRID:AB_2340674 rat cy2; RRID:AB_2340667 rat cy3; RRID: AB_2340672 rat cy5; RRID:AB_2315777 mouse cy3; RRID:AB_2340607 rabbit cy5; RRID:AB_2340365 chicken cy5). Images were acquired on a Leica TCS SP2 laser scanning spectral confocal microscope. Maximal projections of $Z$-stacked images were analyzed with ImageJ software (RRID:SCR_003070). Synaptic markers were quantified as the average intensity and the relative number of pixels per cell area above a threshold value. Thresholds were set such that most of the specific signal was selected. Masks covering the area of interest were created by drawing a line around the transfected cells. The intensity and number of positive pixels normalized to the cell area were averaged in each experimental condition.

Biochemical analysis. Cell cultures were homogenized in lysis buffer (50 mm Tris- $\mathrm{HCl}$, pH 7.4, $100 \mathrm{~mm} \mathrm{NaCl}, 5 \mathrm{~mm} \mathrm{MgCl}, 1 \%$ Triton X-100, 
A
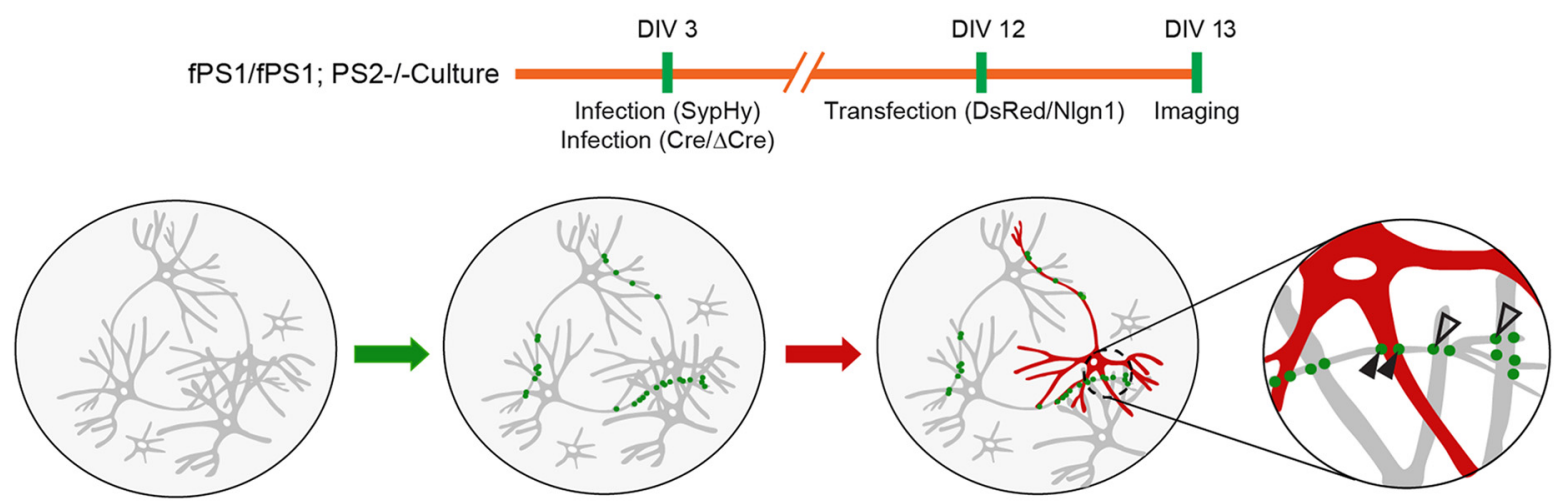

B

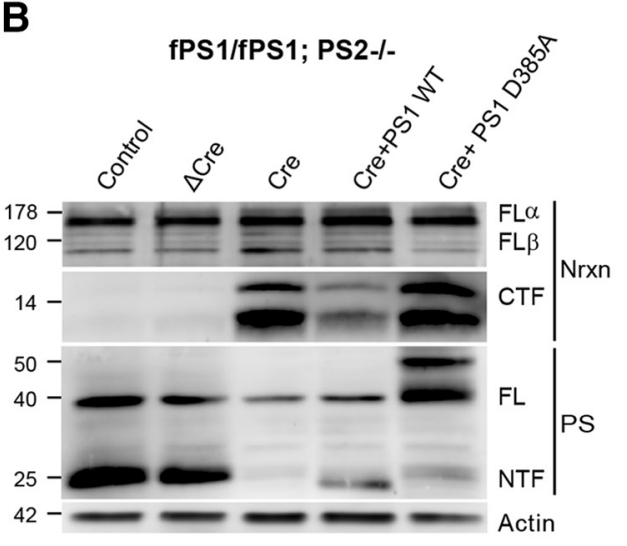

C

D

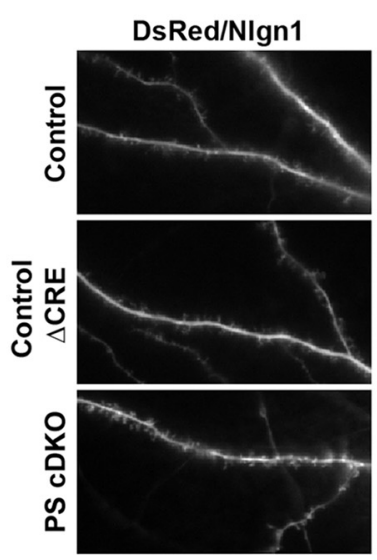

Pre-stimulus
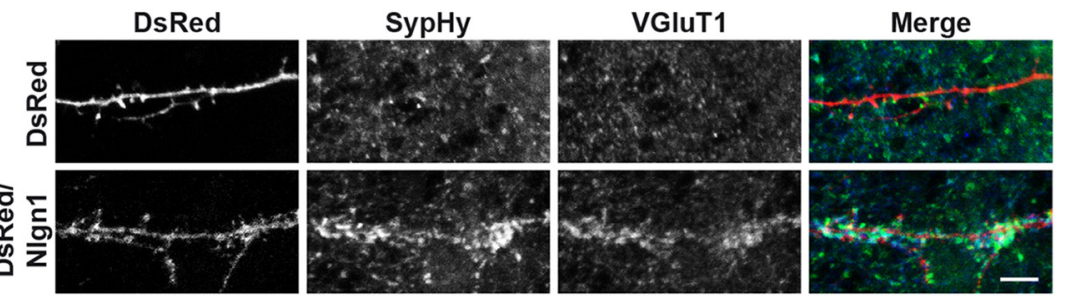

E

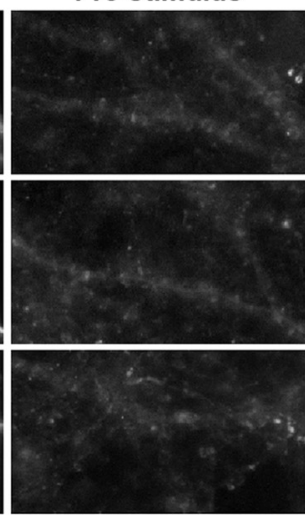

40 AP
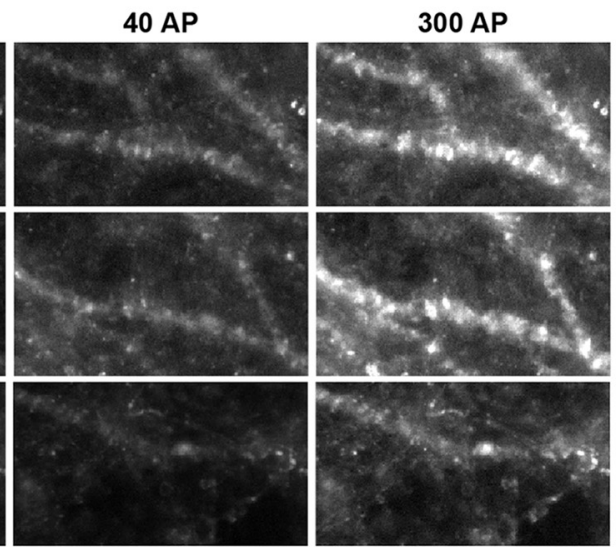

Post-stimulus
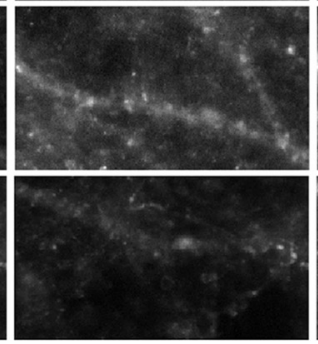

F

G
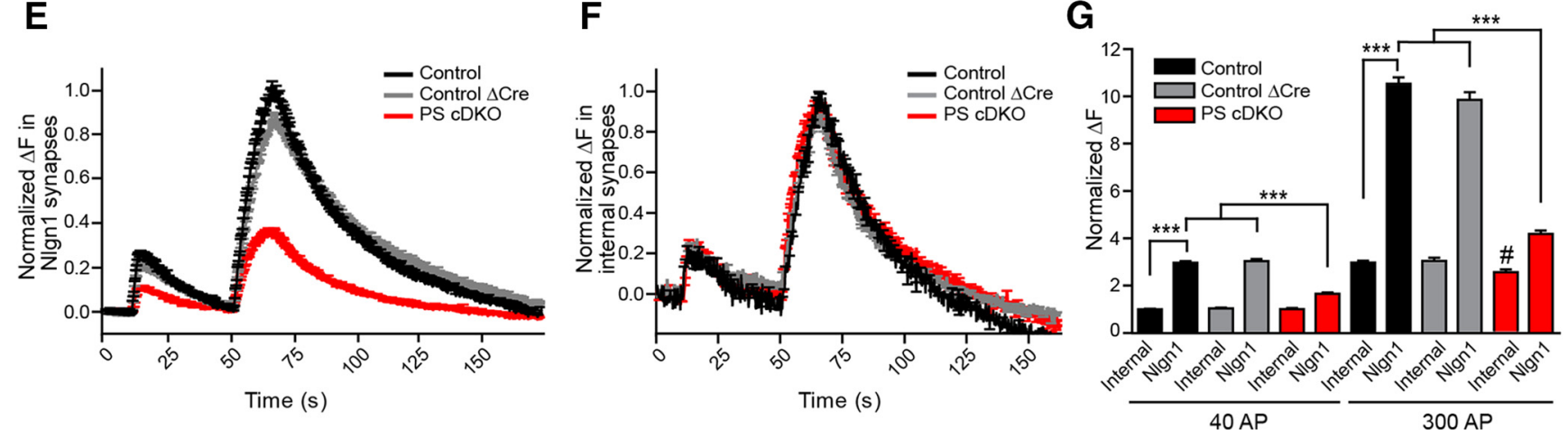

Figure 1. Inhibition of presynaptic release at Nlgn1 synapses of PS CDK0 neurons. $A$, Experimental design. fPS1/fPS1; PS2 ${ }^{-1-}$ hippocampal neurons were infected with lentivirus expressing SypHy alone or double infected with $\Delta$ Cre or Cre, followed by cotransfection of HA-tagged Nlgn 1 and DsRed. The same population of axons forms synaptic puncta in Nlgn1 expressing dendrites (filled arrowheads) and in nonexpressing Nlgn1 synapses (open arrowheads), which represent an internal control. B, Expression of Nrxn-CTF in PS CDKO hippocampal neurons. Lysates from fPS1/fPS1; PS2 ${ }^{-1-}$ cultures (control) and from cultures infected with $\Delta$ (re, Cre, or double infected with Cre and PS1 or PS1 D385A were incubated with the (Figure legend continues.) 
and $0.1 \%$ SDS) containing a protease inhibitor mixture (Roche). When indicated, cells were treated with GM6001 (Calbiochem) and $\mathrm{N}-[\mathrm{N}-(3,5-$ difluorophenacetyl-L-alanyl]-S-phenylglycine t-butyl ester (DAPT; SigmaAldrich). For immunoprecipitation experiments, cell lysates or conditioned media were incubated with the hemagglutinin (HA) antibody. The immune complexes were isolated with Protein-G Sepharose and resolved in SDS-PAGE gels. Western blot experiments of immunoprecipitates or lysates containing equal-protein loading were performed using the following primary antibodies: rat anti-HA (1:500), mouse anti-CASK (1: 200), chicken anti cyto-Nrxn (1:200), rabbit anti-Nrxn 1/2/3 against the common cytoplasmic tail (1:500; Synaptic Systems; RRID:AB_10697815), rabbit anti-PS 1 N-terminal (1-65) (1:5000; Merck Millipore; RRID: AB_10682152) and rabbit anti- $\alpha$ actin (1:1000; Sigma-Aldrich; RRID: AB_476693). Immunoreactivity was detected with secondary antibodies conjugated with horseradish peroxidase (1:5000; Jackson ImmunoResearch; RRID:AB_2313567 rabbit; RRID:AB_2339290 chicken; RRID:AB_2338128 rat; RRID:AB_10015289 mouse). Chemiluminescence was developed using Immun-Star WesternC Kit (Bio-Rad) or SuperSignal West Femto Maximum Sensitivity Substrate (Thermo Fisher Scientific) on an ImageQuant LAS4000 Mini (GE Healthcare Life Sciences).

Cell imaging. Imaging experiments in hippocampal neurons expressing synaptic probes were performed as described previously (Rabaneda et al., 2014). Briefly, cultures were perfused with extracellular solution containing the following (in mM): $2.5 \mathrm{KCl}, 119 \mathrm{NaCl}, 2 \mathrm{CaCl}_{2}, 2 \mathrm{MgCl}_{2}, 25$ HEPES, and 30 glucose, pH 7.4, for SypHy or $2.5 \mathrm{KCl}, 136 \mathrm{NaCl}, 2 \mathrm{CaCl}_{2}$, $1.3 \mathrm{MgCl}_{2}, 10$ HEPES, and 10 glucose, $\mathrm{pH} 7.4$, for SyGCamP3; mixtures also contained $0.01 \mathrm{~mm} \mathrm{CNQX}$ and $0.05 \mathrm{~mm}$ APV (Ascent Scientific). Electrical field stimulation was delivered with a 2100 isolated pulse stimulator (AM Systems). Fluorescence images were acquired on an Axio Observer.A1 inverted microscopy (Zeiss) with a $63 \times$ Zeiss Plan-Apochromat oil-immersion objective. Images were captured with a Hamamatsu Orca $R^{2}$ camera controlled by Micro-Manager software (Edelstein et al., 2010, RRID: SCR_000415). Neurons were stimulated with 5 trains of 1, 3, and 5 APs for SyGCaMP3 experiments or with 40 and 300 APs for SypHy experiments, both delivered at $20 \mathrm{~Hz}$ using a biphasic pulse of $10 \mathrm{~mA}$ and $5 \mathrm{~ms}$ of duration. Time-lapse images were acquired at $4 \mathrm{fps}$ (SyGCaMP3) or 1 fps (SypHy), $672 \times 512$ pixels (binned $2 \times 2$ ), with an exposure time of $100 \mathrm{~ms}$. Routinely, three to five fields of datasets were recorded from each coverslip. Responding synapses were selected manually as regions of interest (ROIs) with the Time Series Analyzer plugin of ImageJ software (RRID:SCR_014269). The standard size of the selected ROIs was $1 \mu \mathrm{m}^{2}$. Background noise within each experiment was quantified as the average intensity of five nonresponding regions and subtracted from each responding ROI. The increase in fluorescence $(\delta F)$ was calculated in the selected ROIs.

Experimental design and statistical analysis. Experimental design for imaging approaches using fluorescent synaptic probes is summarized in Figures 1, 2, 7, and 8. In Figure 1, $E-G$ (12 experiments obtained from 2 independent cultures), $n=355$ for internal control synapses, $n=407$ for

$\leftarrow$

(Figure legend continued.) indicated antibodies. The cyto-Nrxn antibody recognizes the cytoplasmic tail in intact Nrxns and in Nrxn-CTF proteins. C, Corecruitment of SynaptophysinpHluorin (SypHy) and VGluT1 by Nlgn1 in transfected neurons. D, Images showing SypHy fluorescence from hippocampal cultures expressing SypHy alone (control) or together with $\Delta$ Cre or Cre (PS CDKO neurons). SypHy response was elicited with 40 and $300 \mathrm{APS}$, as indicated. Note that the increased SypHy response in Nlgn1-expressing dendrites was decreased in PS CDKO neurons. $\boldsymbol{E}-\boldsymbol{G}$, Kinetics $(\boldsymbol{E}, \boldsymbol{F})$ and peak amplitudes $(\boldsymbol{G})$ of normalized SypHy fluorescence in Nlgn1-induced $(\boldsymbol{E})$ and internal synapses $(\boldsymbol{F})$. Data were normalized to the maximum SypHy fluorescence value in control cultures $(\boldsymbol{E}, \boldsymbol{F})$ or to the peak SypHy fluorescence value elicited with $40 \mathrm{APs}$ at internal synapses of control cultures (G) (normalized $\Delta F$ at $40 \mathrm{APs}$ : internal control synapses $1.0 \pm 0.025$; internal $\Delta$ Cre synapses $1.032 \pm 0.038$; internal PS CDKO synapses $1.009 \pm 0.047 ; \mathrm{Nlgn} 1$ synapses $2.963 \pm 0.074 ; \mathrm{Nlgn} 1 \Delta$ Cre synapses $3.036 \pm 0.088 ; \mathrm{Nlgn} 1 \mathrm{PS}$ CDK0 synapses $1.658 \pm 0.055$; normalized $\Delta F$ at 300 APs: internal control synapses $2.975 \pm$ 0.085 ; internal $\Delta$ Cre synapses $3.048 \pm 0.130$; internal PS CDKO synapses: $2.509 \pm 0.110$; Nlgn1 synapses $10.535 \pm 0.272$, Nlgn $1 \Delta$ Cre synapses $9.856 \pm 0.320$, Nlgn 1 PS CDKO synapses $4.183 \pm 0.141$, \#p $<0.05,{ }^{* * *} p<0.001$, Kruskal-Wallis followed by the post hoc Dunn's test). Scale bars, $5 \mu \mathrm{m}$. Error bars indicate SEM.
Nlgn 1 synapses, $n=200$ for internal $\Delta$ Cre synapses, $n=322$ for Nlgn 1 $\Delta$ Cre synapses, $n=271$ for internal PS cDKO synapses, and $n=443$ for Nlgn1 PS cDKO synapses. In Figure 2, $B-D$ (21 experiments obtained from 3 independent cultures), $n=841$ for internal control synapses, $n=$ 854 for Nlgn 1 synapses, $n=700$ for internal DAPT synapses, and $n=684$ for DAPT Nlgn1 synapses. In Figure 2, $F-H$ (10 experiments obtained from 2 independent cultures), $n=383$ for internal control synapses, $n=$ 387 for Nlgn1 synapses, $n=332$ for internal PS1 D385A synapses, and $n=348$ for PS1 D385A Nlgn1 synapses. In Figure 7C (23 experiments from 3 independent cultures), $n=210$ for control synapses, $n=196$ for HA-Nrxn-CTF $\Delta$ PDZ synapses, and $n=196$ for HA-Nrxn-CTF synapses. In Figure $8 B$ (6 experiments from 2 independent cultures), $n=55$ for internal control synapses, $n=133$ for Nlgn 1 synapses, $n=45$ for internal $\Delta$ Cre synapses, $n=61$ for Nlgn $1 \Delta$ Cre synapses, $n=64$ for internal PS cDKO synapses, and $n=142$ for Nlgn 1 PS cDKO synapses. In Figure $8 D$ (13 experiments from 2 independent cultures), $n=133$ for control synapses, $n=135$ for HA-Nrxn-CTF $\Delta$ PDZ synapses, and $n=97$ for HA-Nrxn-CTF synapses. In Figure $9 D$ (10 experiments from 2 independent cultures), $n=91$ for control synapses, $n=267$ for HA-Nrxn1- $\beta$ synapses, and $n=213$ for HA-Nrxn1- $\beta \Delta 368-393$ synapses. Statistical analyses were performed with GraphPad Prism software (RRID: SCR_002798). For comparison of two groups, the two-tailed Student's $t$ test or the Mann-Whitney $U$ test were applied. Differences in means across multiple groups were analyzed using one-way ANOVA. When the ANOVA analysis revealed significant differences, the post hoc Bonferroni's test was used for pairwise comparisons. When parametric assumptions were not met, Kruskal-Wallis followed by the post hoc Dunn's test was used. All data are presented as mean \pm SEM.

\section{Results}

PS function is essential for presynaptic release at glutamatergic terminals mediated by Nrxn-Nlgn 1

After the shedding of the extracellular domain of Nrxn isoforms by metalloproteases, Nrxn-CTF are processed by PS/gammasecretase at glutamatergic terminals (Saura et al., 2011). However, the functional consequences of the proteolytic processing of Nrxns at glutamatergic synapses are not known. Specifically, because the cytoplasmic tail of Nrxns regulates neurotransmitter release (Futai et al., 2007; Rabaneda et al., 2014; Aoto et al., 2015), we speculated that accumulation of Nrxn-CTF due to impaired PS function might interfere with synaptic vesicle release. For that, we developed an approach that allows for the analysis of presynaptic release at glutamatergic terminals mediated by the NrxnNlgn1 complex in control and PS cDKO hippocampal neurons (Fig. 1A). PS cDKO neurons were generated by Cre-dependent ablation of PS1 in fPS1/fPS1; PS2 ${ }^{-1-}$ hippocampal cultures. As expected, Nrxn-CTF bands recognized by a cyto-Nrxn antibody accumulated in PS cDKO neurons, but not in cultures infected with an inactive $\Delta$ Cre, by a PS-dependent proteolytic processing (Fig. 1B). Control and PS cDKO hippocampal cultures were infected at 3 days in vitro (DIV) with lentivirus expressing SypHy, a $\mathrm{pH}$-sensitive probe that measures exocytosis/endocytosis of synaptic vesicles (Granseth et al., 2006). After infection, the cultures were cotransfected at 11-12 DIV with DsRed and a N-terminal HA-tagged Nlgn1. Therefore, Nlgn 1 coexpressed in DsReddendrites binds to endogenous Nrxns in contacting axons and the activity of the resulting glutamatergic terminals containing SypHy can be analyzed upon stimulation (Fig. 1C).

Previous reports using electrophysiological recordings in hippocampal slices have shown that Nlgn1 increases release probability by a retrograde mechanism (Futai et al., 2007). Because release probability positively correlates with the size of the readily releasable pool (RRP) of synaptic vesicles, which corresponds to the number of docked vesicles at presynaptic active zones (Murthy and Stevens, 1999; Murthy et al., 2001; Schikorski 
A
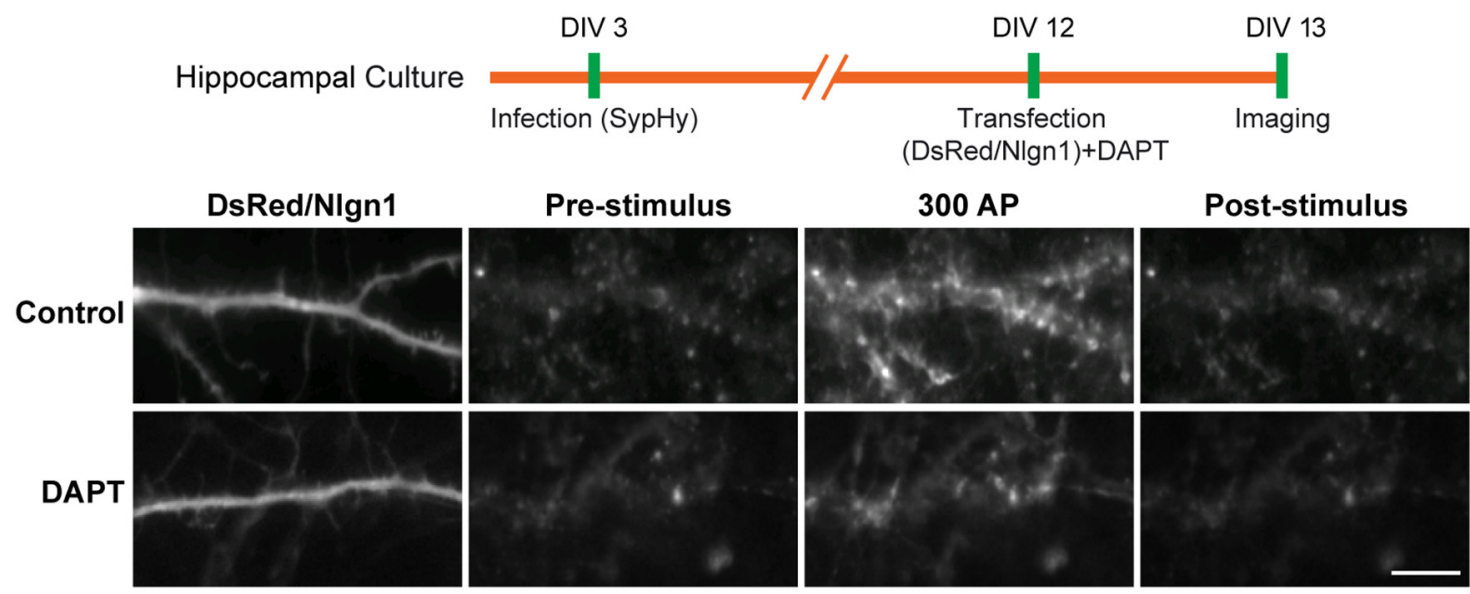

B

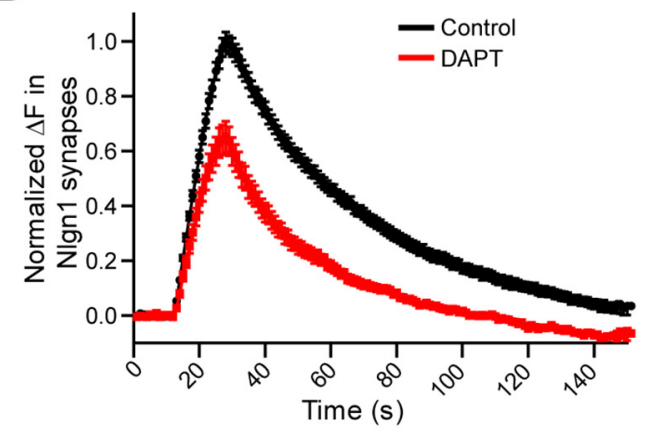

C

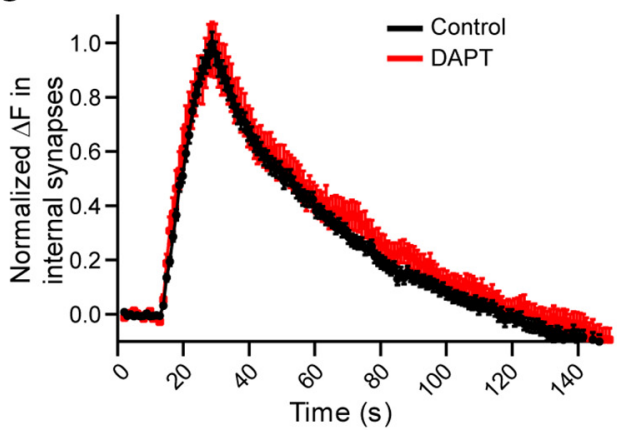

D

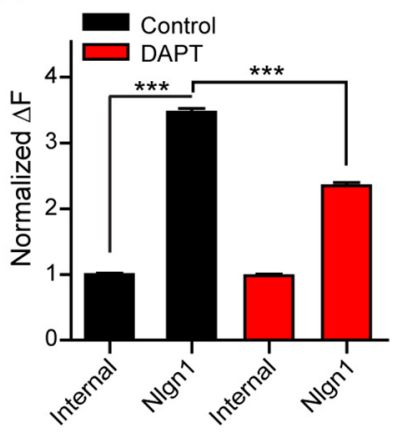

E Hippocampal Culture

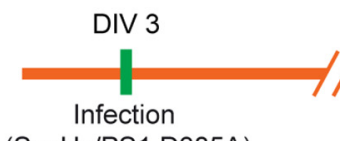

(SypHy/PS1 D385A)

DIV 12 DIV 13
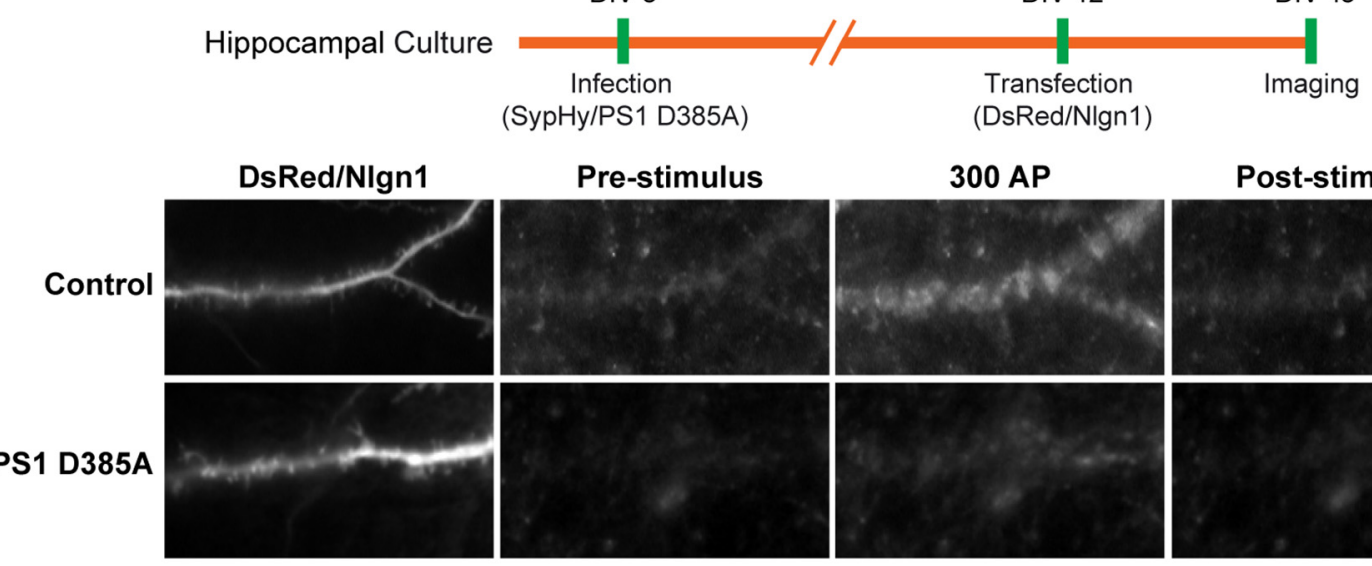

$\mathbf{F}$

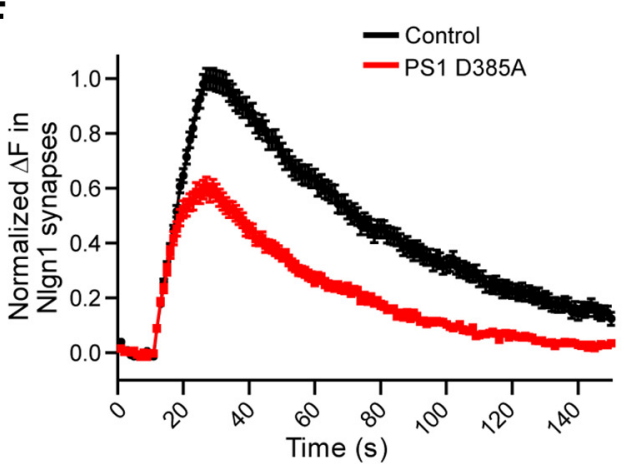

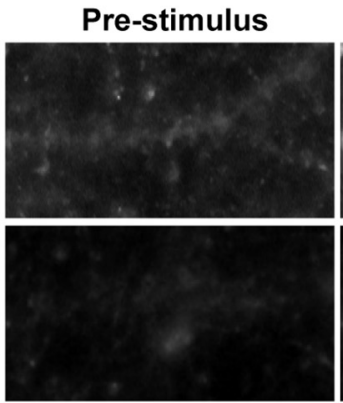

G
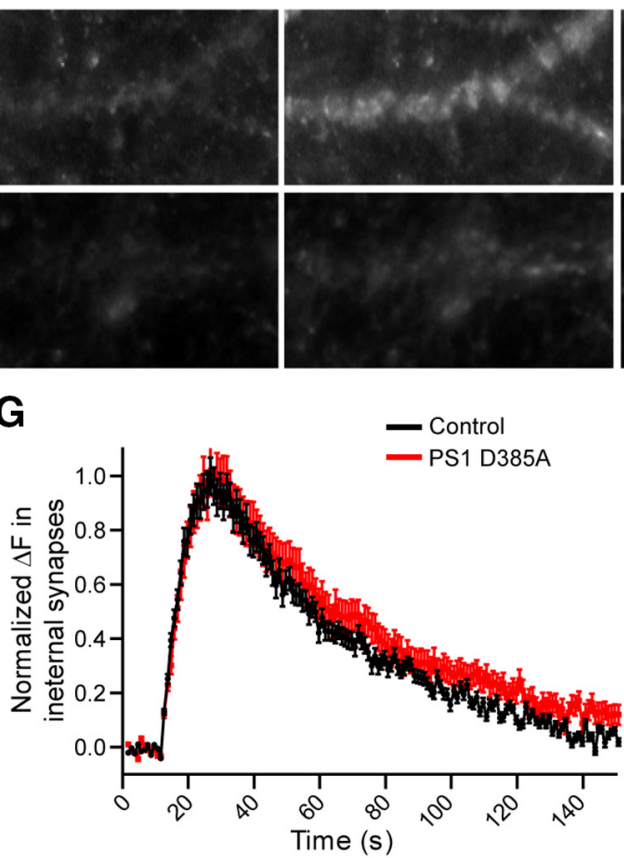

\section{Post-stimulus}
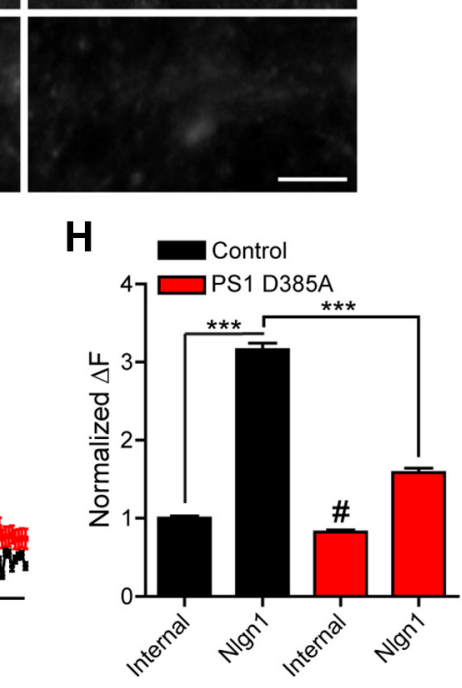

Figure 2. Inhibition of PS proteolytic activity reduces presynaptic release. $A$, Images showing Synaptophysin-pHluorin (SypHy) response at 300 APs of hippocampal neurons incubated with DAPT for $12 \mathrm{~h}$ after cotransfection of DsRed and Nlgn1. $\boldsymbol{B}, \boldsymbol{C}$, Time kinetics of Nlgn1 $(\boldsymbol{B})$ and internal synapses $(\boldsymbol{C})$ in the presence of DAPT. $\boldsymbol{D}$, Peak amplitudes of SypHy fluorescence in internal and Nlgn1-induced synapses in the presence of DAPT (normalized $\Delta$ F: internal control synapses $1.0 \pm 0.023$; internal DAPT synapses $0.98 \pm 0.025$; Nlgn 1 synapses $3.472 \pm 0.054$; DAPT Nlgn1 synapses $2.350 \pm 0.050,{ }^{* * *} p<0.001$, Kruskal-Wallis followed by the post hoc Dunn's test). $\boldsymbol{E}$, Changes in SypHy fluorescence evoked by 300 APs in hippocampal cultures (Figure legend continues.) 
and Stevens, 2001), we measured the RRP with a stimulus train of $40 \mathrm{APs}$ at $20 \mathrm{~Hz}$, followed by 300 APs at the same frequency to mobilize the recycling pool (RP). Presynaptic response at Nlgn1 synapses was compared in each experimental condition with the response of internal synapses, which were identified in the same fields as SypHy-positive puncta not contacting Nlgn1/DsRed transfected dendrites. Interestingly, postsynaptic expression of Nlgn1 in control cultures induced an $\sim 3$-fold increase in presynaptic response at 40 and 300 APs compared with neighboring internal synapses ( $\Delta F$ ratio for Nlgn1/internal synapses at $40 \mathrm{APs}$ : 2.96; $\Delta F$ ratio for Nlgn $1 /$ internal synapses at 300 APs: 3.54) (Fig. $1 D, E, G)$. These data indicated that Nlgn 1 increases the size of the RRP and the RP proportionally at contacting terminals. Remarkably, the positive effect of Nlgn 1 increasing presynaptic release at 40 and 300 APs was strongly reduced in PS CDKO neurons (normalized $\Delta$ Fat 40 APs in Nlgn1 synapses: $2.963 \pm 0.074$; normalized $\Delta F$ at 40 APs in Nlgn 1 PS cDKO synapses: $1.658 \pm 0.055, p<$ 0.0001 , Kruskal-Wallis followed by the post hoc Dunn's test; normalized $\Delta F$ at 300 APs in Nlgn 1 synapses: $10.535 \pm 0.272$; normalized $\Delta F$ at 300 APs in Nlgn1 PS cDKO synapses: $4.183 \pm$ 0.141, $p<0.0001$, Kruskal-Wallis followed by the post hoc Dunn's test; Fig. $1 D, E, G)$. However, presynaptic release at internal synapses was not affected at 40 APs or slightly reduced at 300 APs in PS cDKO neurons (normalized $\Delta F$ at 40 APs in internal control synapses: $1.0 \pm 0.025$; normalized $\Delta F$ at 40 APs in internal PS cDKO synapses: $1.009 \pm 0.047, p>0.999$, Kruskal-Wallis followed by the post hoc Dunn's test; normalized $\Delta F$ at 300 APs in internal control synapses: $2.975 \pm 0.085$; normalized $\Delta F$ at 300 APs in internal PS cDKO synapses: $2.509 \pm 0.110, p=0.048$, Kruskal-Wallis followed by the post hoc Dunn's test; Fig. $1 D, F, G)$. The higher impact of the loss of PS function in Nlgn1 synapses was observed even when presynaptic responses of similar magnitude were compared (40 APs in Nlgn1 synapses vs 300 APs in internal synapses; Fig. 1). These findings suggest a higher susceptibility of Nlgn 1 synapses to the loss of PS function. Moreover, neither Nlgn 1 synapses nor internal synapses were affected in neurons infected with inactive $\Delta C$ re, which was used as a negative control (Fig. 1D-G). These data indicate that Nrxn-Nlgn1 trans-synaptic signaling increases the size of the RRP locally by a mechanism that is affected by the loss of PS function.

\section{PS regulates presynaptic release by a proteolytic mechanism} PS genes have been proposed to regulate cellular signaling pathways by gamma-secretase-dependent and -independent mechanisms (Tu et al., 2006; Hass et al., 2009). To determine whether PS regulates presynaptic release at Nlgn1 synapses by a proteolytic activity or by an alternative mechanism, we inhibited PS/ gamma-secretase proteolytic activity, but not PS expression, using the gamma-secretase inhibitor DAPT ( $1 \mu \mathrm{M})$ or expressing the dominant-negative mutant PS1 D385A, two approaches that induce the accumulation of Nrxn-CTF in hippocampal neurons (Saura et al., 2011). Interestingly, we found that impairment of

\section{$\leftarrow$}

(Figure legend continued.) expressing PS1 D385A. Hippocampal neurons were double infected with lentivirus expressing PS1 D385A and SypHy before transfection of DsRed and NIgn1. F, G, Time kinetics of $\operatorname{Nlgn} 1(\boldsymbol{F})$ and internal synapses $(\boldsymbol{G})$ of cultures expressing PS1 D385A. $\boldsymbol{H}$, Peak amplitudes of SypHy response in internal and Nlgn1-induced synapses expressing PS1 D385A (normalized $\Delta F$ : internal control synapses $1.0 \pm 0.030$; internal PS1 D385A synapses $0.825 \pm$ 0.027; Nlgn1 synapses $3.159 \pm 0.082 ;$ PS1 D385A Nlgn1 synapses $1.585 \pm 0.057$, \#p $<0.01$, ${ }^{* * *} p<0.0001$, Kruskal-Wallis followed by the post hoc Dunn's test). Data were normalized to the maximum SypHy fluorescence value of control cultures $(\boldsymbol{B}, \boldsymbol{C}, \boldsymbol{F}, \boldsymbol{G})$, or to the peak SypHy fluorescence of internal control synapses $(\boldsymbol{D}, \boldsymbol{H})$. Scale bars, $5 \mu \mathrm{m}$. Error bars indicate SEM.
PS/gamma-secretase activity in hippocampal neurons strongly reduced presynaptic release at 300 APs in Nlgn 1 synapses in both cultures treated with DAPT (normalized $\Delta F$ in Nlgn 1 synapses: $3.472 \pm 0.054$; normalized $\Delta F$ in DAPT Nlgn 1 synapses: $2.350 \pm$ $0.050, p<0.0001$, Kruskal-Wallis followed by the post hoc Dunn's test) and cultures expressing PS1 D385A (normalized $\Delta F$ in Nlgn1 synapses: $3.159 \pm 0.082$; normalized $\Delta F$ in PS1 D385A Nlgn 1 synapses: $1.585 \pm 0.057, p<0.0001$, Kruskal-Wallis followed by the post hoc Dunn's test; Fig. 2). Presynaptic release at internal synapses was not affected by DAPT treatment (normalized $\Delta F$ in internal synapses of control neurons: $1.0 \pm 0.023$; normalized $\Delta F$ in internal synapses of DAPT neurons: $0.98 \pm$ $0.025, p>0.999$, Kruskal-Wallis followed by the post hoc Dunn's test) or slightly reduced by the expression of PS1 D385A (normalized $\Delta F$ in internal synapses of control neurons: $1.0 \pm 0.030$; normalized $\Delta F$ in internal synapses of PS1 D385A neurons: $0.825 \pm 0.027, p=0.0072$, Kruskal-Wallis followed by the post hoc Dunn's test; Fig. 2). These data obtained with genetic or chemical inhibitors of PS/gamma-secretase activity recapitulated the effects observed in PS cDKO neurons, suggesting that loss of PS proteolytic activity at Nrxn-Nlgn 1 synapses accounts for the inhibitory effect on presynaptic release.

\section{PS function is dispensable for the recruitment of synaptic vesicles induced by Nlgn 1}

Expression of Nlgn 1 increases the recruitment of synaptic vesicles and the density of presynaptic terminals in contacting axons (Scheiffele et al., 2000; Dean et al., 2003). Therefore, we investigated whether PS activity is also required for the synaptogenic function of Nlgn1 or if loss of PS function specifically inhibits presynaptic release. Quantitative analysis of SypHy, used as a synaptic vesicle marker, by confocal microscopy showed a $>2$-fold increase in SypHy intensity at Nlgn 1 synapses compared with internal synapses in control neurons (normalized SyPhy intensity in internal synapses: $1.0 \pm 0.007$; normalized SyPhy intensity in Nlgn1 synapses: $2.194 \pm 0.027, p<0.0001$, Mann-Whitney $U$ test; Fig. 3). However, inhibition of PS function did not affect the area or the intensity of presynaptic SypHy recruited at Nlgn 1 synapses in any of the three experimental approaches studied, namely PS cDKO hippocampal neurons (Fig. $3 A-C$ ) and hippocampal cultures treated with DAPT or expressing PS1 D385A (Fig. 3D-I). In agreement with these data, the levels of the endogenous synaptic vesicle marker VGluT1 recruited by Nlgn1 were not affected in PS cDKO neurons or in neurons treated with DAPT (data not shown). Moreover, the levels of transfected Nlgn1 in PS defective neurons were not different from control neurons in any of the different approaches (Fig. 3C,F,I). These data further support that the inhibition in presynaptic release caused by inactivation of PS is not an indirect consequence of reduced synaptic vesicle recruitment or Nlgn 1 expression suggesting a presynaptic mechanism. Moreover, the immunofluorescence signal obtained with a cyto-Nrxn antibody was increased at Nlgn1 synapses of PS cDKO neurons compared with Nlgn1 synapses of control cultures (Fig. $3 J, K$ ). These data suggested local accumulation of Nrxn-CTF at glutamatergic terminals of PS defective neurons, in agreement with previous results (Saura et al., 2011). The levels of CASK, a synaptic scaffolding protein that can interact with a PDZ-binding domain in the cytoplasmic tail of Nrxns (Butz et al., 1998), were increased significantly at Nlgn 1 synapses of PS cDKO neurons (Fig. 3J,L). These results indicate that the proteolytic activity of the PS/gamma-secretase complex plays a selective role in the release of synaptic vesicles, which can be dissected apart 
A

fPS1/fPS1; PS2-I-

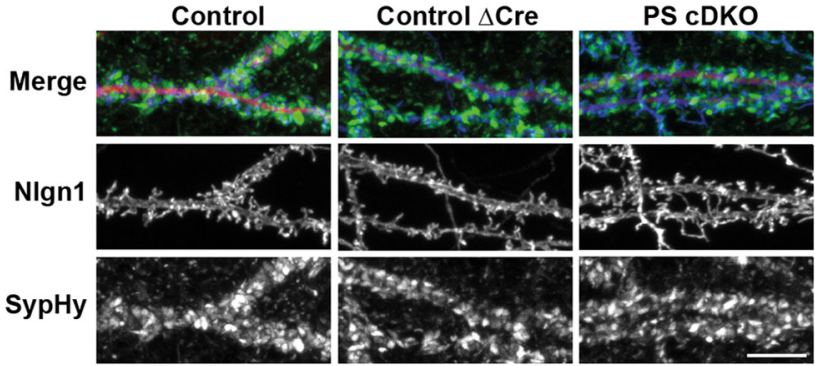

B

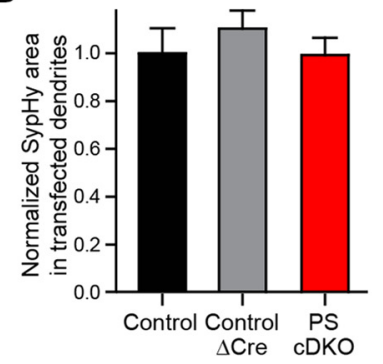

C

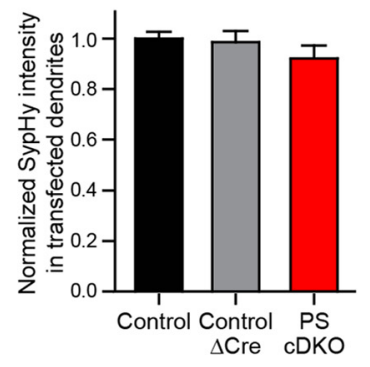

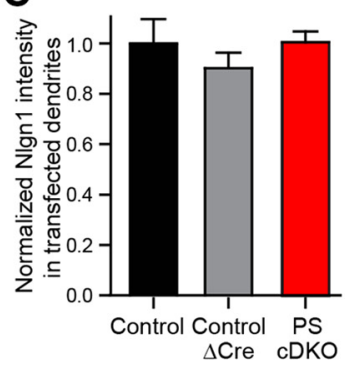

D

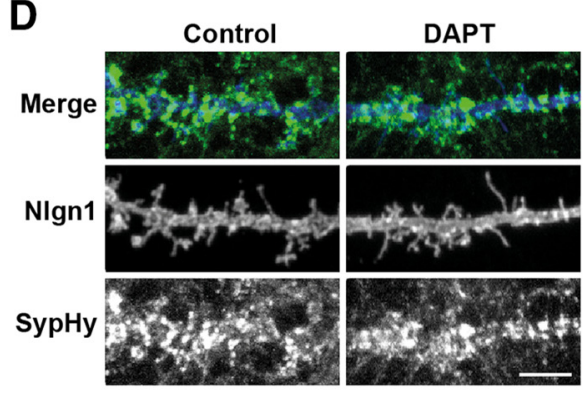

G

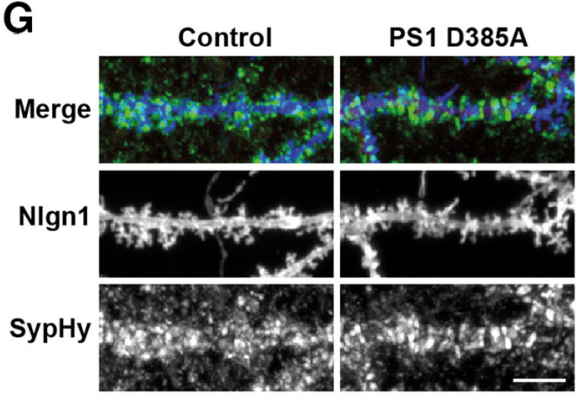

E

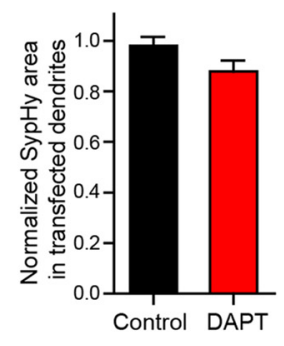

H

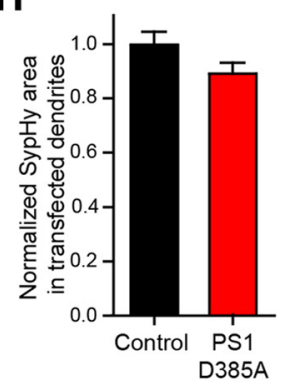

F

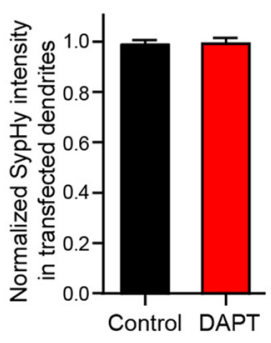

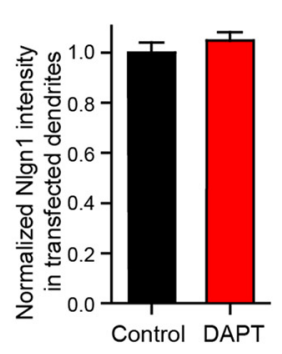

I
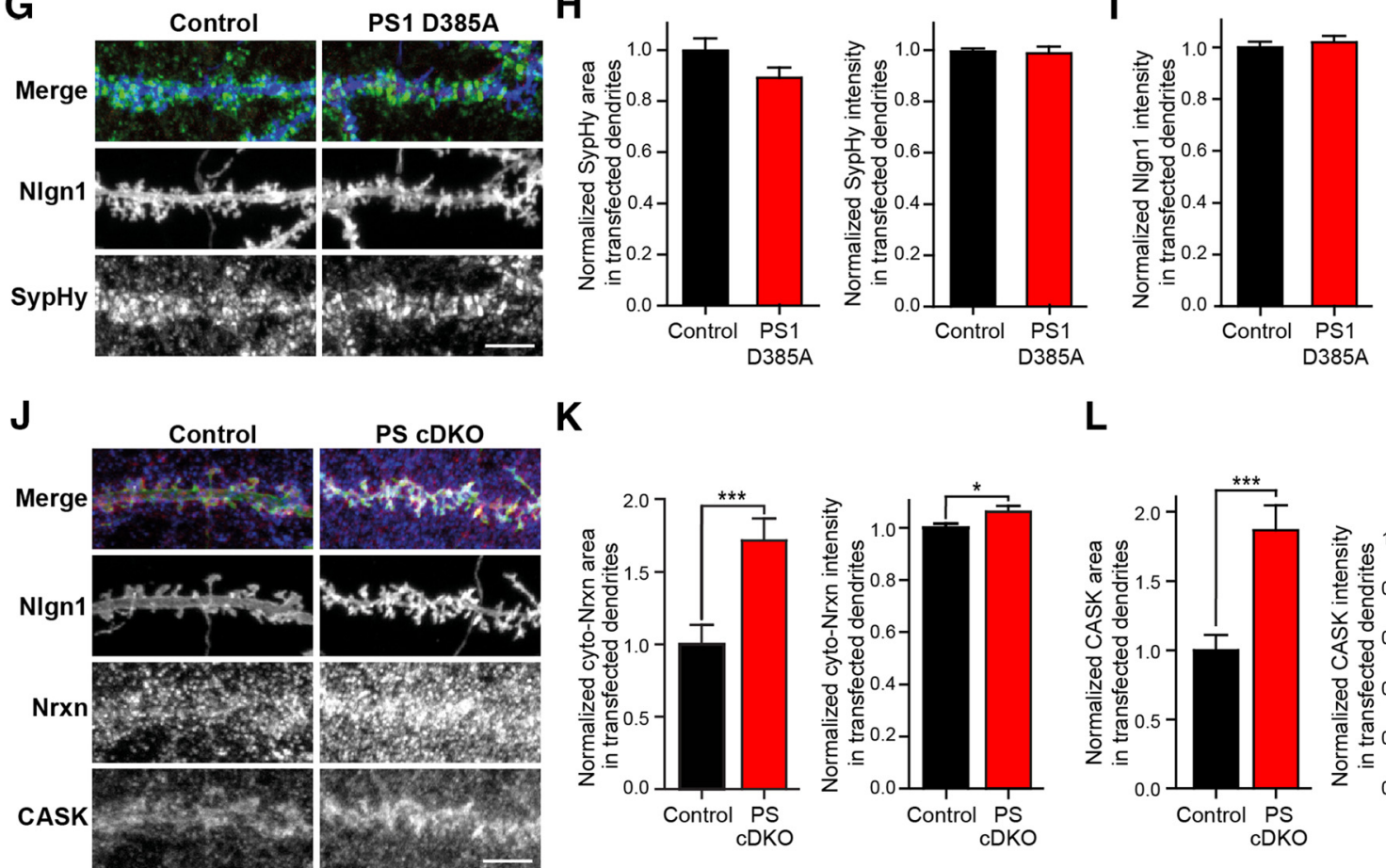

K
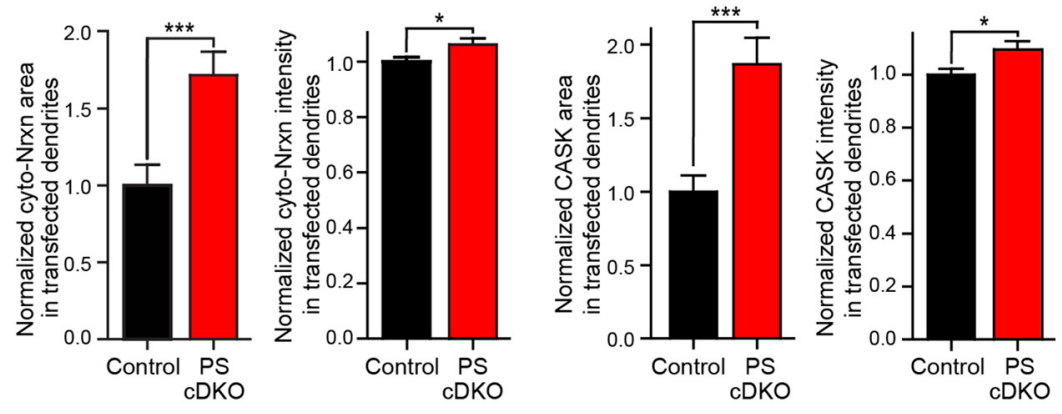

Figure 3. Distribution of synaptic markers at synapses induced by NIgn1 in hippocampal neurons with impaired PS function. $\boldsymbol{A}-\boldsymbol{C}$, Immunofluorescence staining and quantifications of the synaptic vesicle-resident marker Synaptophysin-pHluorin (SypHy) and Nlgn1 in PS CDKO hippocampal neurons (normalized SypHy area: control 1.0 \pm 0.105 ; control $\Delta$ Cre 1.103 \pm 0.076 ; PS CDKO $0.992 \pm 0.073, p>0.05$, one-way ANOVA followed by the post hoc Bonferroni's test; normalized SypHy intensity: control $1.0 \pm 0.025$; (Figure legend continues.) 
A

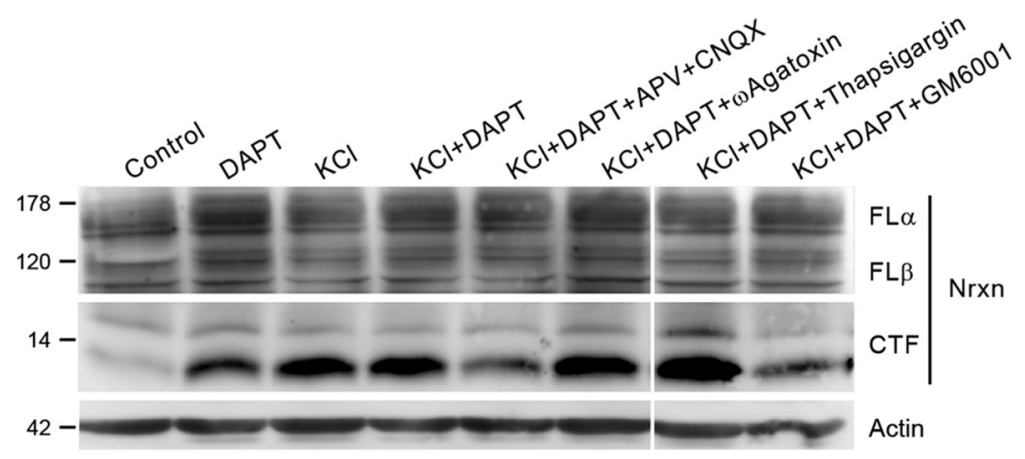

B

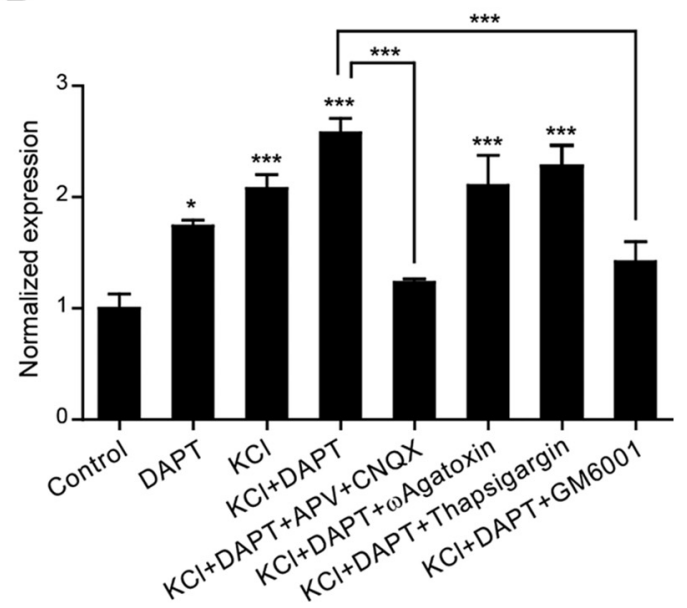

Figure 4. Activity-dependent proteolytic processing of Nrxns. $A$, Western blot experiments of hippocampal lysates incubated with $50 \mathrm{~mm} \mathrm{KCl} \mathrm{for} 30$ min and treated in the presence of the indicated inhibitors. Nrxns were detected with a cyto-Nrxn antibody, as indicated. $\boldsymbol{B}$, Graph shows quantification of normalized data (control 1.0 $\pm 0.127 ;$ DAPT 1.739 $\pm 0.051 ; \mathrm{KCl} 2.077 \pm 0.123$; $\mathrm{KCl}+\mathrm{DAPT} 2.576 \pm 0.130 ; \mathrm{KCl}+\mathrm{DAPT}+\mathrm{APV}+\mathrm{CNQX} 1.234 \pm 0.030 ; \mathrm{KCl}+\mathrm{DAPT}+\omega$-agatoxin-IVA $2.103 \pm 0.273 ; \mathrm{KCl}+\mathrm{DAPT}+$ thapsigargin $2.280 \pm 0.187 ; \mathrm{KCl}+\mathrm{DAPT}+\mathrm{GM} 60011.419 \pm$ $0.179, n=8,{ }^{*} p<0.05,{ }^{* * *} p<0.001$, one-way ANOVA followed by the post hoc Bonferroni's test). Error bars indicate SEM.

from the recruitment of synaptic vesicles and the synapse formation activity induced by Nlgn1.

\section{Activity-dependent processing of Nrxns}

The previous results suggested the generation of a PS/gammasecretase substrate that abnormally accumulates at Nrxn-Nlgn1 synapses with impaired PS activity and ultimately mediates the inhibition in presynaptic release. The accumulation of NrxnCTF at glutamatergic terminals makes it a strong candidate to mediate the inhibitory effect of PS in neurotransmitter release. It has been reported previously that depolarization with $\mathrm{KCl}$ or incubation with glutamate induces the generation of Nrxn-CTF in cultured neurons (Bot et al., 2011). To further analyze the mechanism responsible for the activity-dependent proteolytic processing of Nrxns, we depolarized hippocampal cultures with $50 \mathrm{~mm} \mathrm{KCl}$ for $30 \mathrm{~min}$ and evaluated the levels of Nrxn-CTF by

$\leftarrow$

(Figure legend continued.) control $\Delta$ Cre $0.986 \pm 0.043$; PS CDK0 $0.921 \pm 0.050, p>0.05$, Kruskal-Wallis followed by the post hoc Dunn's test; normalized Nlgn1 intensity: control $1.0 \pm$ 0.095; control $\Delta$ Cre $0.902 \pm 0.060$; PS CDK0 $1.004 \pm 0.041, p>0.05$, Kruskal-Wallis followed by the post hoc Dunn's test). Nlgn1 was detected with an HA antibody. Only Nlgn1 intensity was analyzed due to the high dendritic area covered by overexpressed NIgn1. D-F, Immunofluorescence staining of SypHy and $\mathrm{NIgn} 1$ and quantifications in hippocampal neurons incubated with DAPT (normalized SypHy area: control $1.0 \pm 0.035$; DAPT $0.896 \pm 0.044, p=$ 0.076 , Student's $t$ test; normalized SypHy intensity: control $1.0 \pm 0.017$; DAPT $1.004 \pm 0.022$, $p=0.878$, Student's $t$ test; normalized Nlgn1 intensity: control $1.0 \pm 0.040$; DAPT $1.046 \pm$ $0.034, p=0.402$. Mann-Whitney U test). $\mathbf{G}-\boldsymbol{I}$, Immunofluorescence signal of SypHy and NIgn1 and quantifications in hippocampal neurons expressing PS1 D385A (normalized SypHy area: control 1.0 $\pm 0.076 ;$ PS1 D385A $0.825 \pm 0.058, p=0.075$, Student's t test; normalized SypHy intensity: control 1.0 $\pm 0.011 ;$ PS1 D385A $0.966 \pm 0.019, p=0.133$, Student's $t$ test; normalized Nlgn1 intensity: control 1.0 \pm 0.034 ; PS1 D385A $1.029 \pm 0.031, p=0.538$, Student's $t$ test). J, Immunofluorescence experiments with CASK and cyto-Nrxn antibodies showing increased signals at Nlgn1 synapses of PS CDKO neurons. $K, L$, Graphs showing quantifications of the relative area and mean intensity of cyto-Nrxn $(\boldsymbol{K})$ and CASK $(\boldsymbol{L})$ fluorescent signals at Nlgn1 synapses of control ( $\Delta \mathrm{Cre}$ ) and PS CDKO neurons (normalized Nrxn area: control $\Delta$ Cre $1.0 \pm$ 0.132 ; PS CDK0 1.716 $\pm 0.153, p=0.001$, Student's $t$ test; normalized Nrxn intensity: control $\Delta$ Cre $1.0 \pm 0.015$; PS CDK0, $1.061 \pm 0.022, p=0.029$, Mann-Whitney $U$ test; normalized CASK area: control $\Delta$ Cre $1.0 \pm 0.109 ;$ PS CDK0 $1.869 \pm 0.178, p=0.0002$, Mann-Whitney $U$ test; normalized CASK intensity: control $\Delta$ Cre $1.0 \pm 0.021$; PS CDK0 1.094 $\pm 0.031, p=0.033$, Mann-Whitney $U$ test). Data from $15-25$ experiments were obtained from two to three independent cultures. Scale bars, $5 \mu \mathrm{m} .{ }^{*} p<0.05,{ }^{* * *} p<0.001$. Error bars indicate SEM.
Western blot. We confirmed that stimulation with $\mathrm{KCl}$ resulted in a $>2$-fold increase in Nrxn-CTF levels compared with nonstimulated cultures (Fig. 4). Inhibition of the PS/gamma-secretase complex with DAPT $(1 \mu \mathrm{M})$ further increased the levels of Nrxn-CTF in cultures stimulated with $\mathrm{KCl}$, indicating activity of the PS/ gamma-secretase complex during synaptic stimulation. Importantly, we found that incubation with the broad metalloprotease inhibitor GM6001 $(2.5 \mu \mathrm{M})$ abolished the KCl-induced accumulation of Nrxn-CTF (Fig. 4). These data suggest that the cleavage of Nrxn ectodomain by metalloproteases initiates the activitydependent production of Nrxn-CTF, which is subsequently processed by PS. We next tested the effect of several antagonists in the activity-dependent generation of Nrxn-CTF. Inhibition of sarco-ER calcium ATPase (SERCA) activity with thapsigargin (4 $\mu \mathrm{M})$, which results in depletion of calcium stores at the ER, or blockage of voltage-dependent calcium-channels with $\omega$-agatoxinIVA (100 nM) did not affect the activity-dependent accumulation of Nrxn-CTF (Fig. 4). However, blocking NMDA and AMPA receptors with APV $(50 \mu \mathrm{M})$ and CNQX $(10 \mu \mathrm{M})$ abolished the accumulation of Nrxn-CTF induced by activity (Fig. 4). These experiments indicate that NMDA/AMPA receptor signaling is required to exscind the ectodomain of Nrxns, leading to the generation of Nrxn-CTF, which is cleared form glutamatergic terminals by a PS/ gamma-secretase activity.

\section{Identification of the cleavage site that generates Nrxn-CTF}

To identify the residues responsible for the generation of NrxnCTF, we produced deletions in the juxtamembrane region of human HA-Nrxn1- $\beta$, a known Nlgn1 presynaptic receptor, and analyzed the generation of the ectodomain and Nrxn-CTF fragments (Fig. 5A). Immunoprecipitates of conditioned media from transfected HEK293T cells showed that increasing the size of deletions within residues $368-385$ produced the release of HANrxn $1-\beta$ ectodomains of decreasing size, suggesting that the localization of the cleavage site was further downstream (Fig. 5B). Furthermore, a larger deletion comprising residues 368-393 inhibited the release of HA-Nrxn1- $\beta$ ectodomain and increased the levels of the mature protein in cell lysates (Fig. $5 B$ ). In agreement with a sequential proteolytic processing, the generation of NrxnCTF, both basal and accumulated in presence of DAPT, was 
A

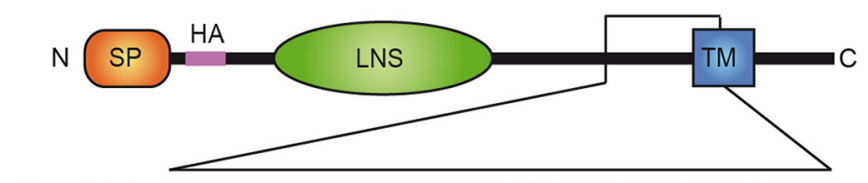

Nrxn- 1 beta DIDPCEPSSGGLANPTRAGGREPYPGSAEVIRESSSTTGMVV $\triangle$ 368-372 DIDPCEPSS-----PTRAGGREPYPGSAEVIRESSSTTGMVV $\triangle$ 368-378 DIDPCEPSS-----------REPYPGSAEVIRESSSTTGMVV $\triangle$ 368-385 DIDPCEPSS------------------AEVIRESSSTTGMVV

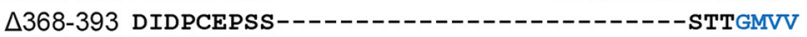

B
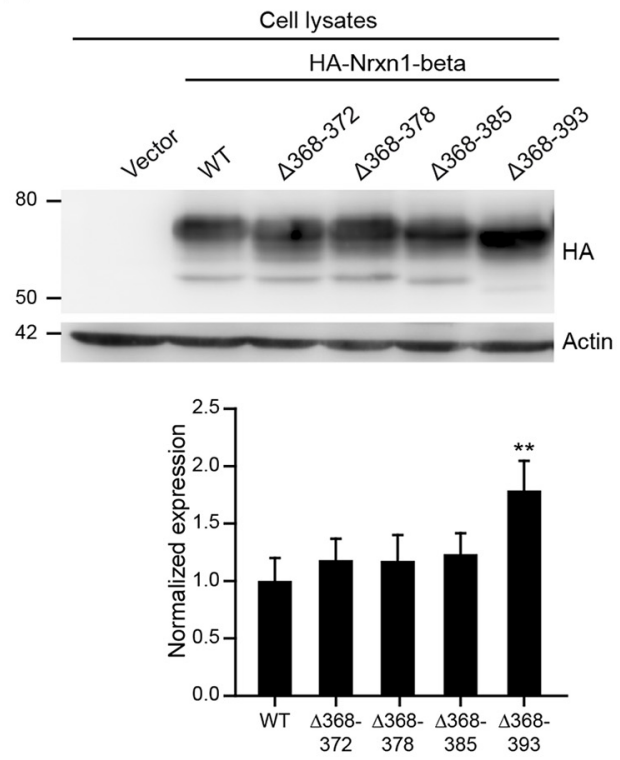
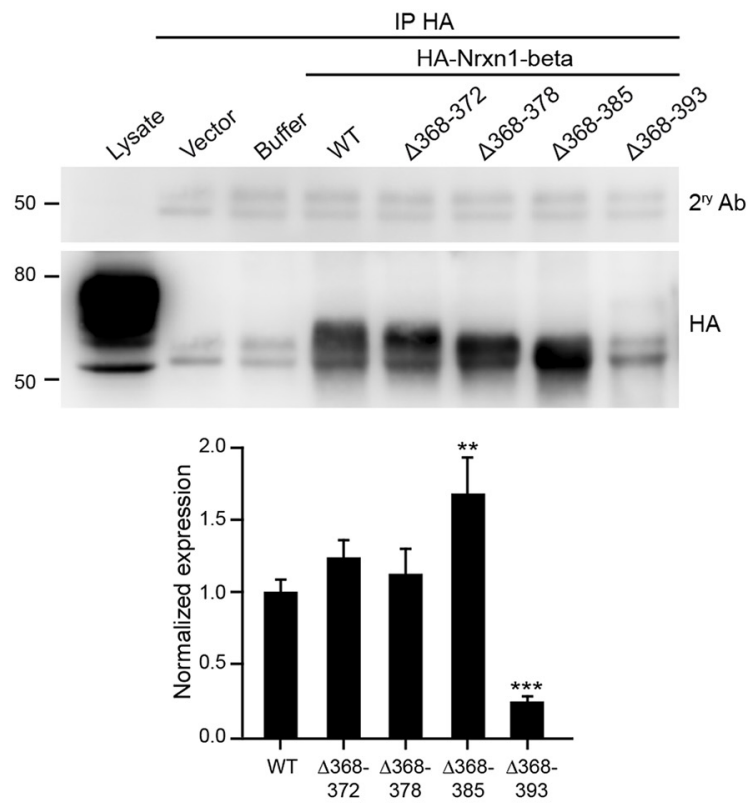

C HA-Nrxn1-beta
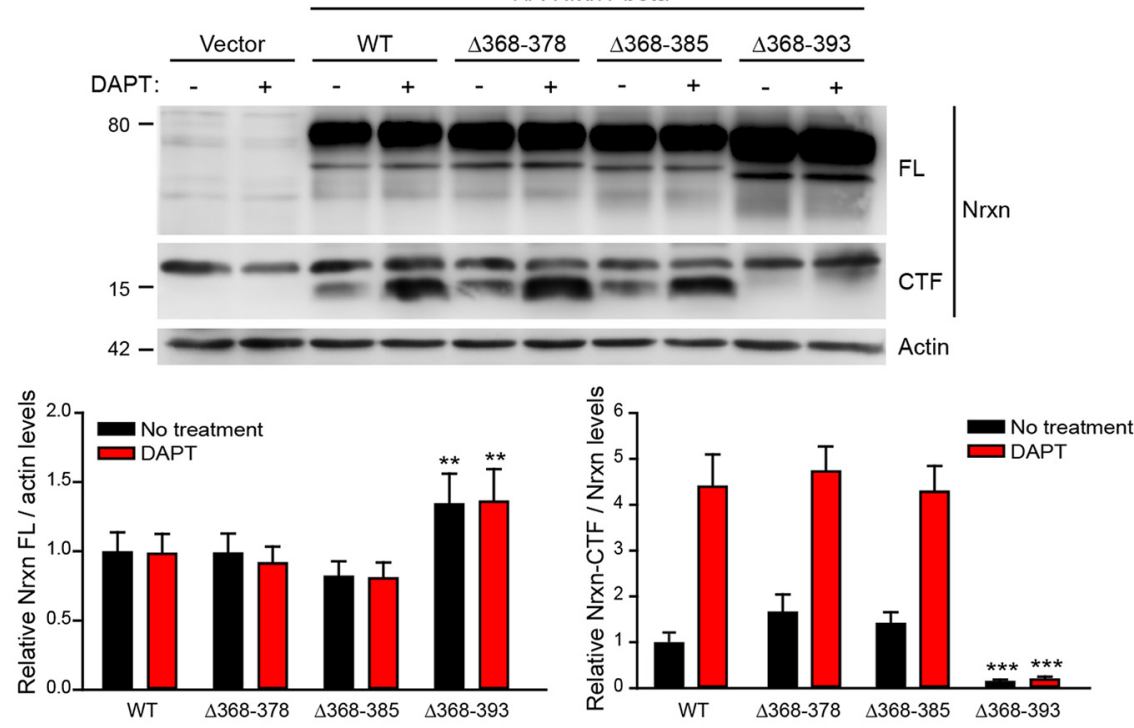

D

NRXN1:

NRXN2:

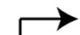

NRXN3:

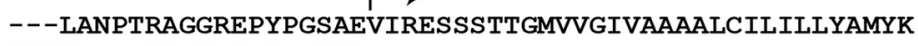

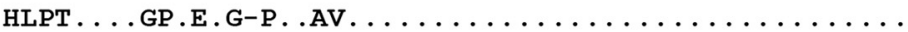

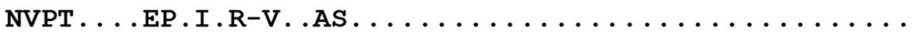

YRNRDEGSYHVDESRNYISNSAQSNGAVVKEKQPSSAKSSNKNKKNKDKEYYV

$\ldots \ldots \ldots$. . Q . . . . . . . . . . . .

$\ldots \ldots \ldots$. . . . . . . . . . TLM....Q.S.GH.KQ.....

Figure 5. Proteolytic processing of Nrxn1- $\beta$ deletion mutants. A, Protein sequences of deletion mutants generated within the juxtamembrane region of human Nrxn1- $\beta$. SP, Signal peptide; LNS, laminin/Nrxn/sex-hormone binding protein domain; $\mathrm{TM}$, transmembrane region. Wild-type protein and each deletion mutant were labeled with an N-terminal $\mathrm{HA}$-tag. $\boldsymbol{B}$, Western blot experiments with an anti-HA antibody of cell lysates or HA immunoprecipitates from conditioned media of HEK293T cells transfected with the indicated construct (cell lysates: WT $1 \pm 0.132 ; \Delta 368-3721.184 \pm$ $0.130 ; \Delta 368-3781.173 \pm 0.152 ; \Delta 368-3851.234 \pm 0.123 ; \Delta 368-3931.789 \pm 0.171,{ }^{* *} p<0.01$, Kruskal-Wallis followed by the posthocDunn's test; HA-immunoprecipitates: (Figurelegend continues.) 
A

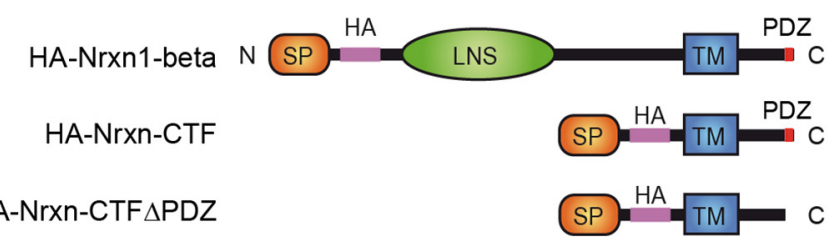

B

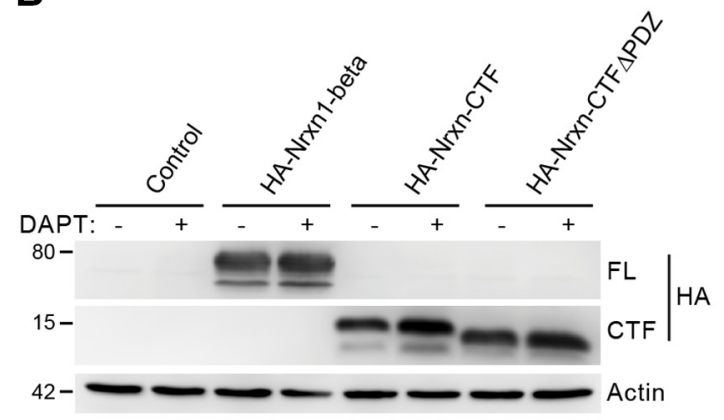

C
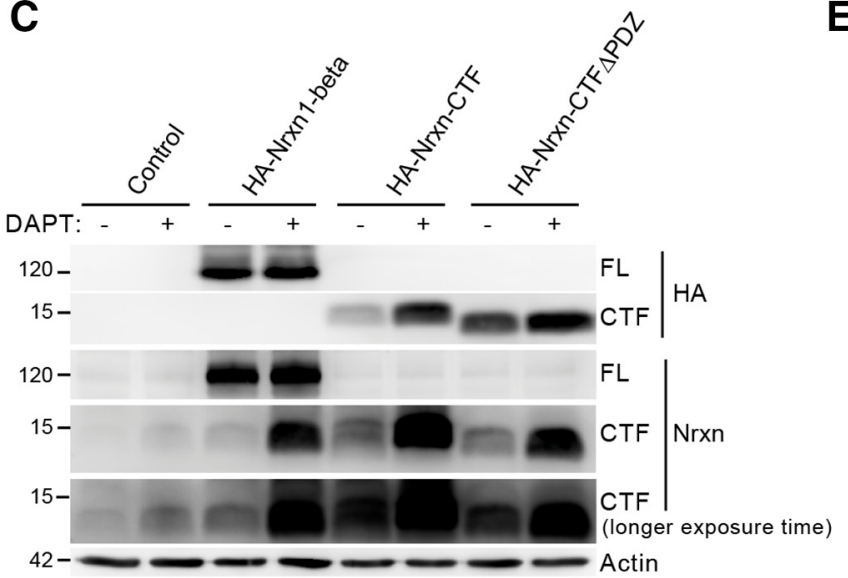

D

E
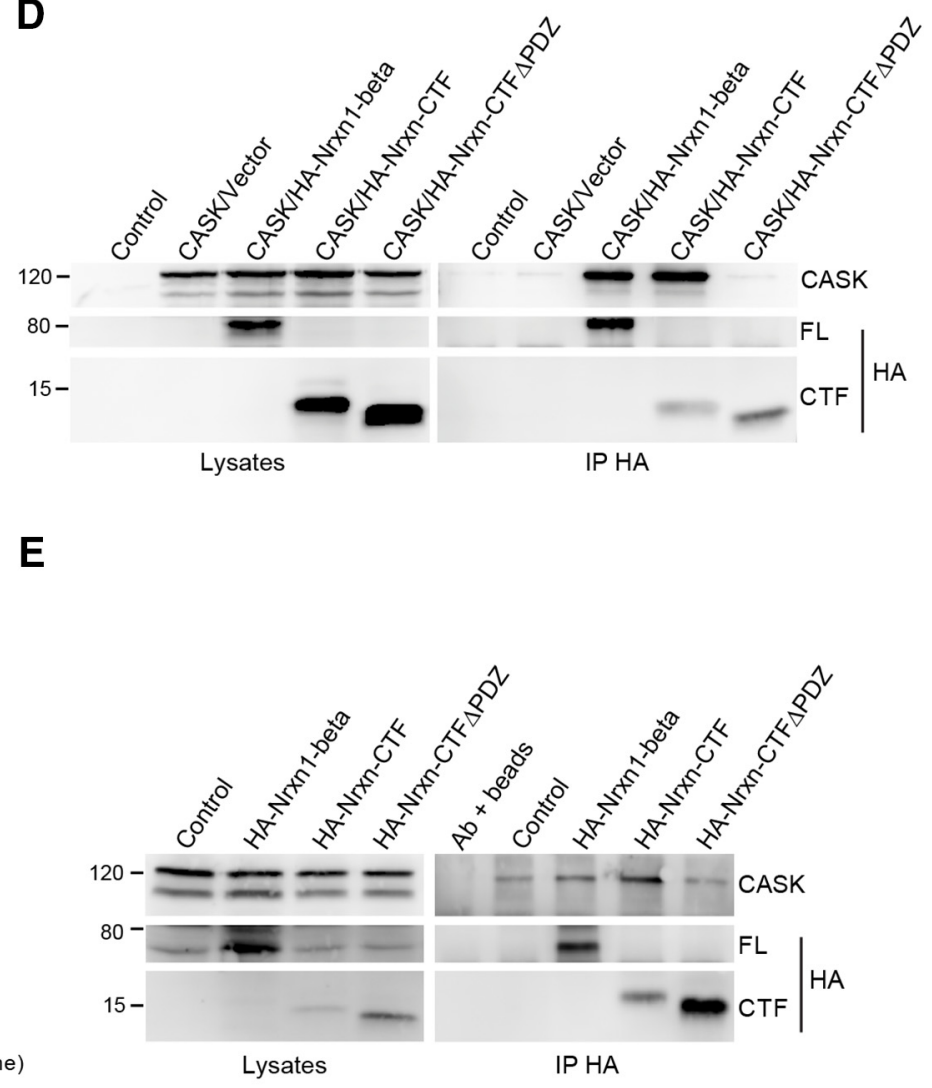

Figure 6. Processing of HA-Nrxn-CTF proteins by PS. A, Schematic drawings showing HA-Nrxn-CTF and HA-Nrxn-CTF $\triangle P D Z$ constructs compared with Nrxn1- $\beta$. PDZ labels the last three residues that form a PDZ-binding domain. $\boldsymbol{B}$, Western blot of transfected HEK293T cells in the presence of DAPT. C, Lysates of hippocampal neurons infected with HA-Nrxn1- $\beta$, HA-Nrxn-CTF or HA-NrxnCTF $\triangle P D Z$ and incubated with DAPT were immunoblotted with HA and cyto-Nrxn antibodies, as indicated. The decreased mobility observed for HA-Nrxn-CTF compared with Nrxn-CTF is likely due to the insertion of the HA tag. $\boldsymbol{D}$, Lysates and HA immunoprecipitates of HEK293T cells coexpressing CASK and empty vector, HA-Nrxn-CTF, or HA-Nrxn-CTF $\Delta$ PDZ. HA-Nrxn1- $\beta$ was coexpressed as a positive control. The isolated HA complexes were immunoblotted with CASK and HA antibodies, as indicated. E, Hippocampal neurons expressing HA-Nrxn-CTF, HA-Nrxn-CTF $\triangle$ PDZ, or HA-Nrxn1- $\beta$ by lentiviral infections were immunoprecipitated with HA antibodies and the presence of CASK and HA proteins analyzed by Western blot.

$\leftarrow$

(Figure legend continued.) WT $1 \pm 0.036 ; \Delta 368-3721.240 \pm 0.050 ; \Delta 368-3781.122 \pm$ $0.081 ; \Delta 368-3851.662 \pm 0.108, \Delta 368-3930.232 \pm 0.006, n=5,{ }^{* *} p<0.01,{ }^{* * *} p<$ 0.001 , Kruskal-Wallis followed by the post hoc Dunn's test). A cell lysate expressing HANrxn1- $\beta$ was included to show the lower molecular weight of the immunoprecipitated ectodomains. C, Detection of Nrxn-CTF in Western blot experiments of transfected HEK293T cells using cyto-Nrxn antibodies (normalized Nrxn FL/ actin levels: WT $1 \pm 0.086 ; \Delta 368-3780.998 \pm$ 0.073; $\Delta 368-3850.830 \pm 0.064 ; \Delta 368-3931.449 \pm 0.132$, WT +DAPT $0.978 \pm 0.089$; $\Delta 368$-378+DAPT $0.926 \pm 0.076 ; \Delta 368-385+$ DAPT $0.812 \pm 0.066 ; \Delta 368-393+$ DAPT $1.492 \pm 0.160, n=5,{ }^{* *} p<0.01$, Kruskal-Wallis followed by the post hoc Dunn's test; normalized Nrxn-CTF/Nrxn levels: WT $1 \pm 0.133 ; \Delta 368-3781.494 \pm 0.186 ; \Delta 368-385$ $1.423 \pm 0.187 ; \Delta 368-3930.118 \pm 0.013 ;$ WT + DAPT $4.428 \pm 0.787 ; \Delta 368-378+$ DAPT $4.753 \pm 0.962 ; \Delta 368-385+$ DAPT $4.354 \pm 0.860 ; \Delta 368-393+$ DAPT $0.194 \pm 0.026, n=$ $5,{ }^{* * *} p<0.001$, Kruskal-Wallis followed by the post hoc Dunn's test). D, Sequence alignment of human Nrxn1-3 proteins showing the high conservation of the shedding site and the predicted Nrxn-CTF. Dots indicate identical amino acids. Arrow indicates V388. strongly inhibited when residues 368-393 were deleted (Fig. 5C). These data revealed a major shedding site within residues $386-$ 393 of Nrxn $1-\beta$ responsible for the release of the ectodomain and the subsequent generation of Nrxn-CTF. Intriguingly, deletion of residues 368-385 increased the levels of HA-Nrxn1- $\beta$ ectodomain in the media, suggesting that residues adjacent to the shedding site negatively regulate Nrxn $1-\beta$ shedding (Fig. $5 B$ ). Sequence alignment showed high homology of the predicted Nrxn-CTF originating from the different Nrxn genes (Fig. 5D).

Next, we generated an HA-Nrxn-CTF expression vector encoding the last $85 \mathrm{C}$-terminal residues of Nrxn1 starting at V388 and tagged at the mature $\mathrm{N}$ terminus with an HA epitope (Fig. $6 A$ ). In parallel, we also generated HA-Nrxn-CTF $\Delta$ PDZ, a mutant lacking the last three $\mathrm{C}$-terminal residues essential in intact Nrxns for the binding to PDZ-synaptic proteins (Butz et al., 1998; Grootjans et al., 2000; Fig. 6A). In Western blot experiments of transfected HEK293T cells, the levels of HA-Nrxn-CTF and HA- 
A
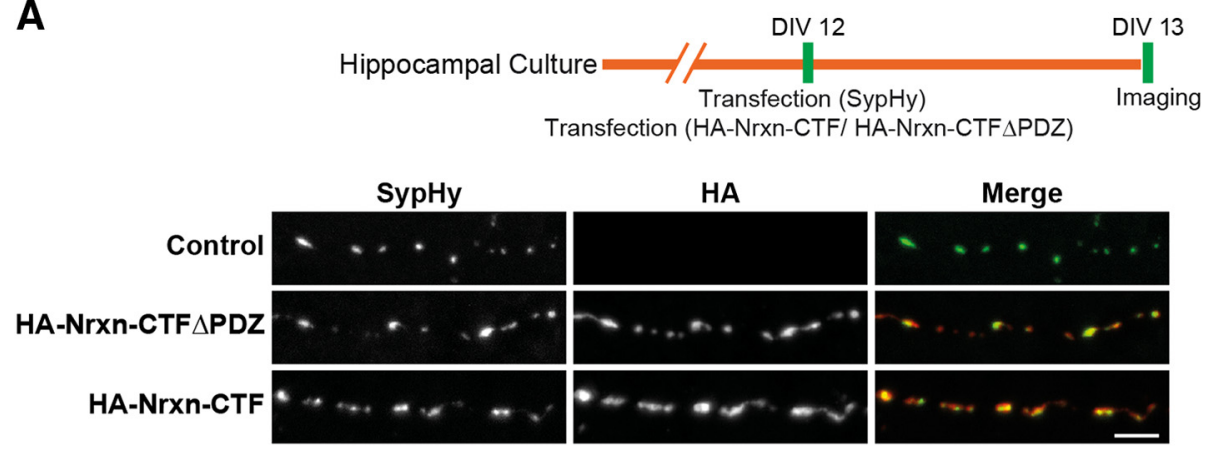

B

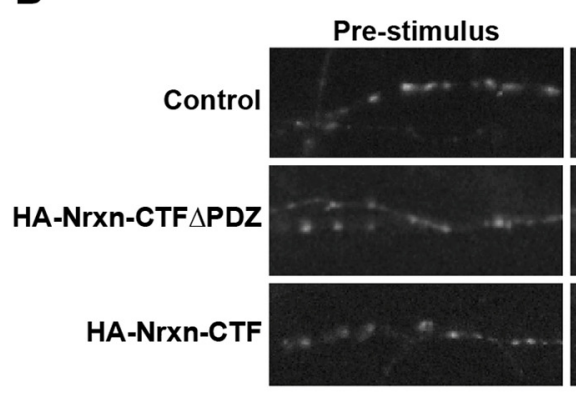

D

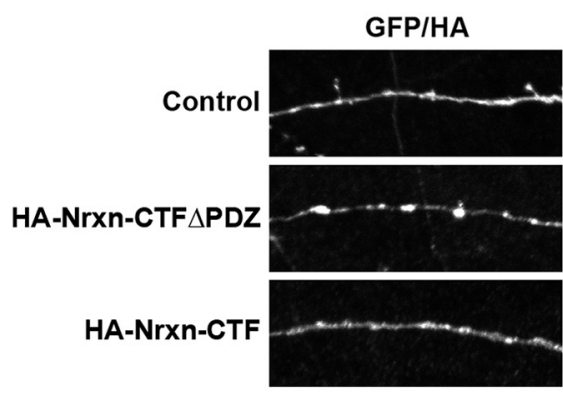

40 AP

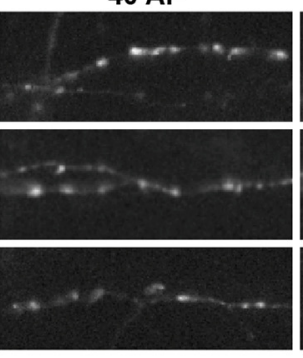

300 AP

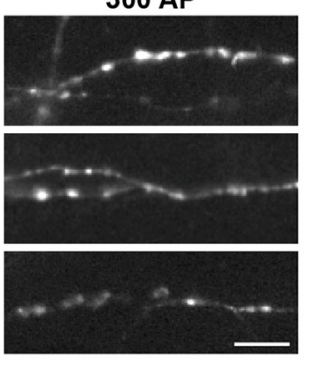

C

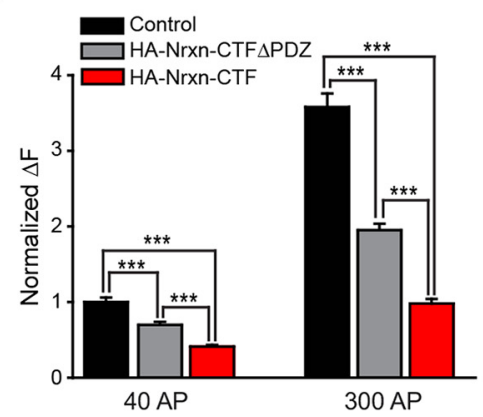

E
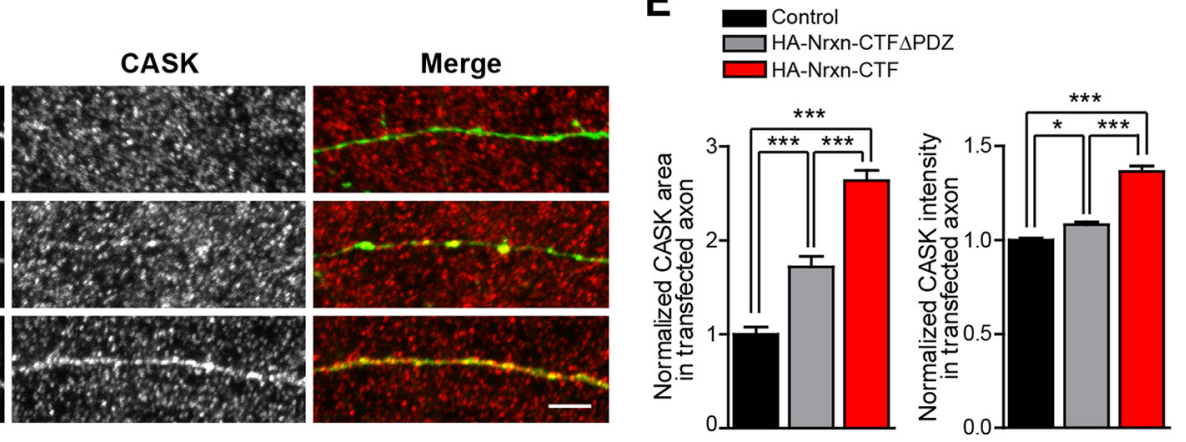

Figure 7. Expression of Nrxn-CTF inhibits presynaptic release. A, HA-Nrxn-CTF or HA-Nrxn-CTF $\Delta$ PDZ were cotransfected with Synaptophysin-pHluorin (SypHy) in hippocampal neurons and stained with HA antibody. Presynaptic terminals expressing SypHy were detected with GFP fluorescence. B, Images showing SypHy response at 40 and 300 APs in axons expressing HA-Nrxn-CTF and HA-Nrxn-CTF $\triangle$ PDZ. C, Peak amplitudes of SypHy fluorescence in axons transfected with HA-Nrxn-CTF and HA-Nrxn-CTF $\triangle$ PDZ compared with control neurons (control synapses at 40 APs $1.0 \pm$ 0.060; HA-Nrxn-CTF synapses at 40 APs $0.411 \pm 0.023$; HA-Nrxn-CTF $\Delta$ PDZ synapses at 40 APs $0.697 \pm 0.041$; control synapses at 300 APs $3.580 \pm 0.178$; HA-Nrxn-CTF synapses at 300 APs $0.980 \pm$ 0.059 ; HA-Nrxn-CTF $\triangle$ PDZ synapses at 300 APs $1.949 \pm 0.085,{ }^{* * *} p<0.001$, Kruskal-Wallis followed by the post hoc Dunn's test). D, Immunofluorescence experiments with HA and CASK antibodies showing CASK accumulation at HA-Nrxn-CTF expressing axons. E, Quantification of CASK staining (normalized CASK area in transfected axons: GFP 1.0 $\pm 0.079 ;$; HA-Nrxn-CTF 2.636 \pm 0.107; HA-Nrxn-CTF $\triangle$ PDZ1.717 $\pm 0.113 ;{ }^{* * *} p<0.001$, one-way ANOVA followed by the post hoc Bonferroni's test. Normalized CASK intensity in transfected axons: GFP 1.0 \pm 0.010 ; HA-Nrxn-CTF $1.365 \pm 0.028$, HA-Nrxn-CTF $\triangle$ PDZ $1.081 \pm 0.015,{ }^{*} p<0.05,{ }^{* * *} p<0.001$, one-way ANOVA followed by the post hoc Bonferroni's test. Data are shown from $26-31$ images from two independent experiments from different cultures. Scale bars, $5 \mu \mathrm{m}$. Error bars indicate SEM.

Nrxn-CTF $\triangle$ PDZ proteins increased in the presence of DAPT, as expected for a PS substrate (Fig. 6B). To confirm the proteolytic processing in neurons, we expressed HA-Nrxn-CTF proteins in hippocampal neurons and analyzed the expression in the presence of DAPT by Western blot using anti-HA and cyto-Nrxn antibodies. HA-Nrxn-CTF showed a similar mobility to NrxnCTF originating from endogenous Nrxns and from the expression of Nrxn1- $\beta$ in the same cultures (Fig. $6 C$ ). Moreover, the finding that HA-Nrxn-CTF were readily detectable in untreated neurons and that their levels further increased upon inhibition of PS activity indicated accumulation of HA-Nrxn-CTF in untreated neurons with otherwise normal PS activity. HA-NrxnCTF, but not HA-Nrxn-CTF $\triangle$ PDZ, coimmunoprecipitated with CASK in heterologous and neuronal cells (Fig. 6D,E), indicating that the PDZ-binding domain of Nrxn-CTF can mediate pro- tein-protein interactions. These data demonstrated that HANrxn-CTF is a PS substrate comparable to endogenous NrxnCTF and provided a molecular tool with which to evaluate the functional consequences of the proteolytic activity of PS on a single substrate, Nrxns.

Nrxn-CTF inhibit presynaptic release

The effect of the accumulation of HA-Nrxn-CTF on presynaptic release was analyzed in hippocampal neurons. We chose transfection experiments to achieve expression in sparse neurons, so that the effect of HA-Nrxn-CTF on presynaptic release could be readily attributable to the presynaptic neuron. Moreover, within the transfected axons, HA-Nrxn-CTF showed localization in synaptic puncta (Fig. 7A), thereby resembling the presynaptic distribution of endogenous Nrxn-CTF. Remarkably, presynaptic 
A
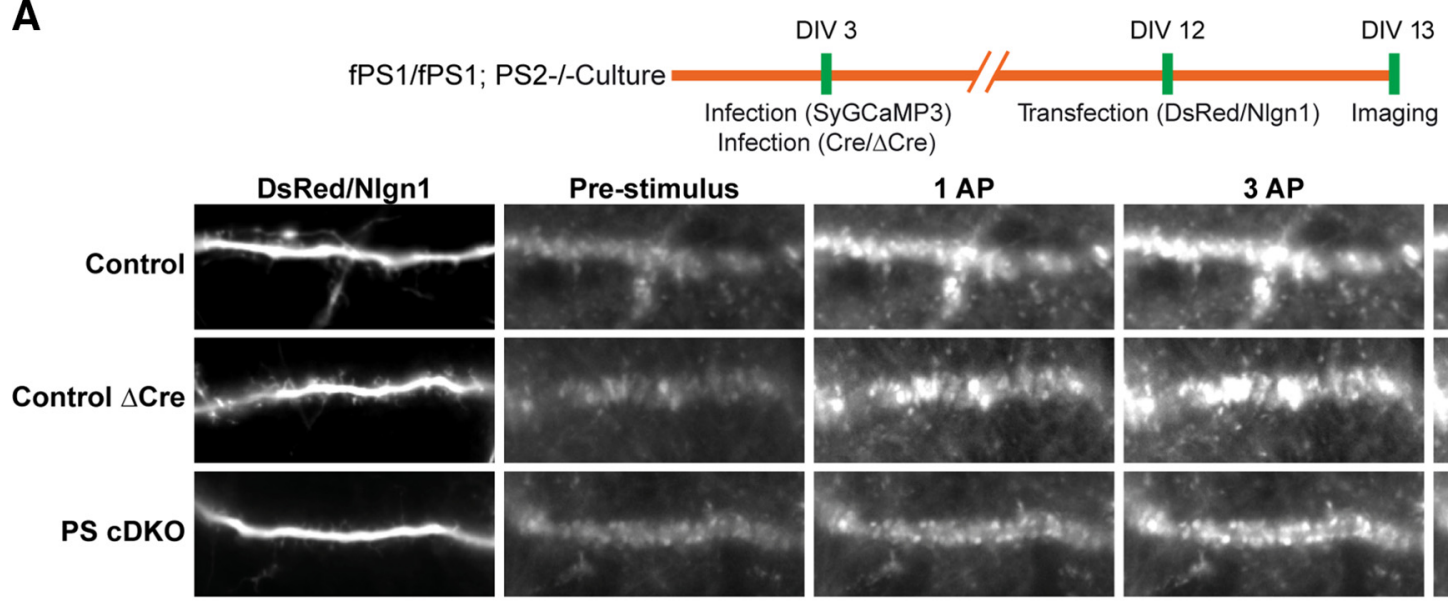

Pre-stimulus
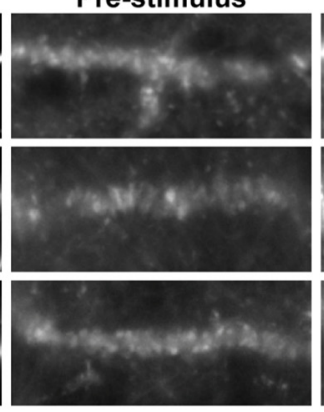

1 AP
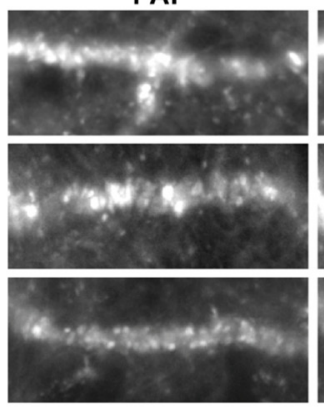

3 AP

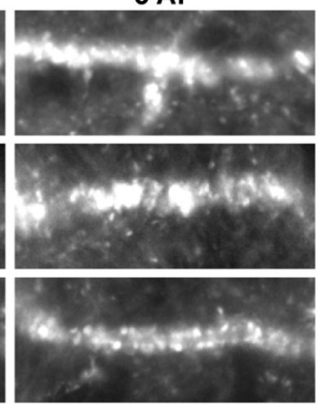

5 AP

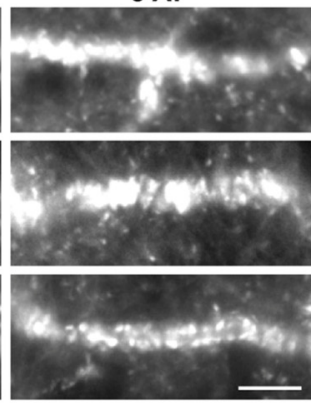

B
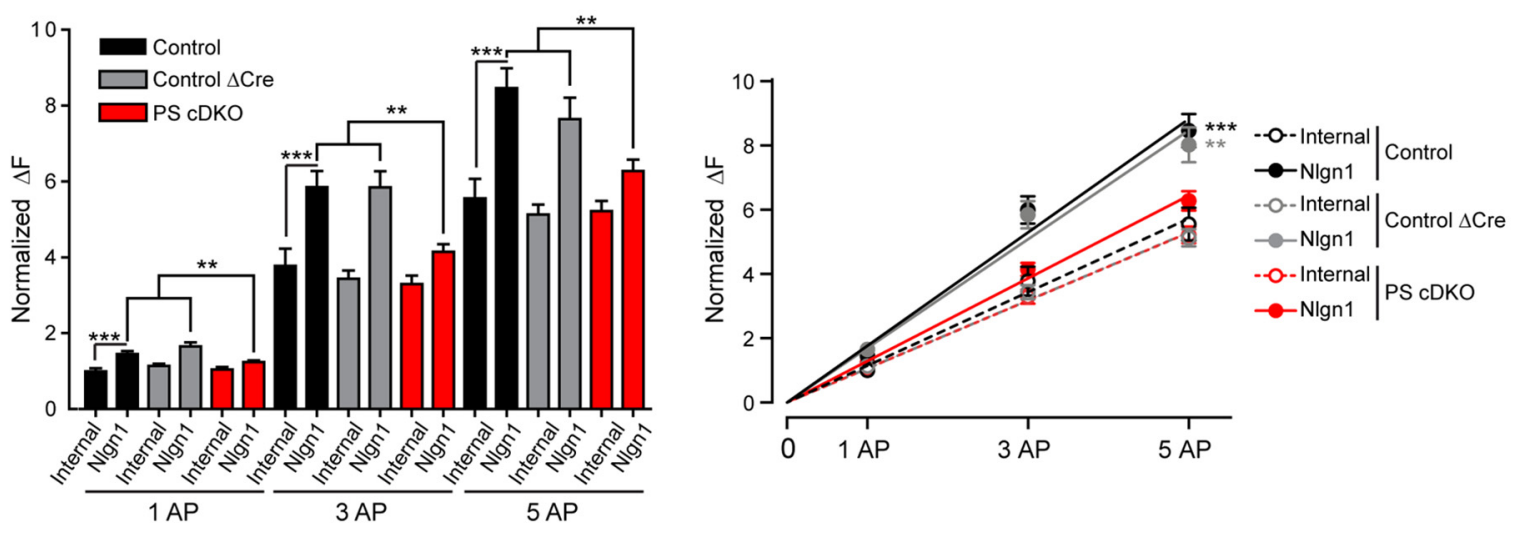

C

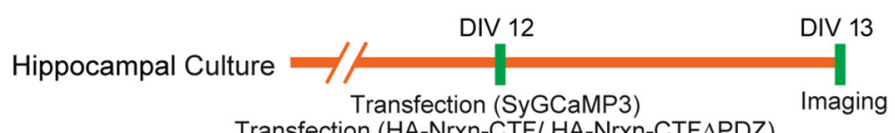
Transfection (HA-Nrxn-CTF/HA-Nrxn-CTFAPDZ)

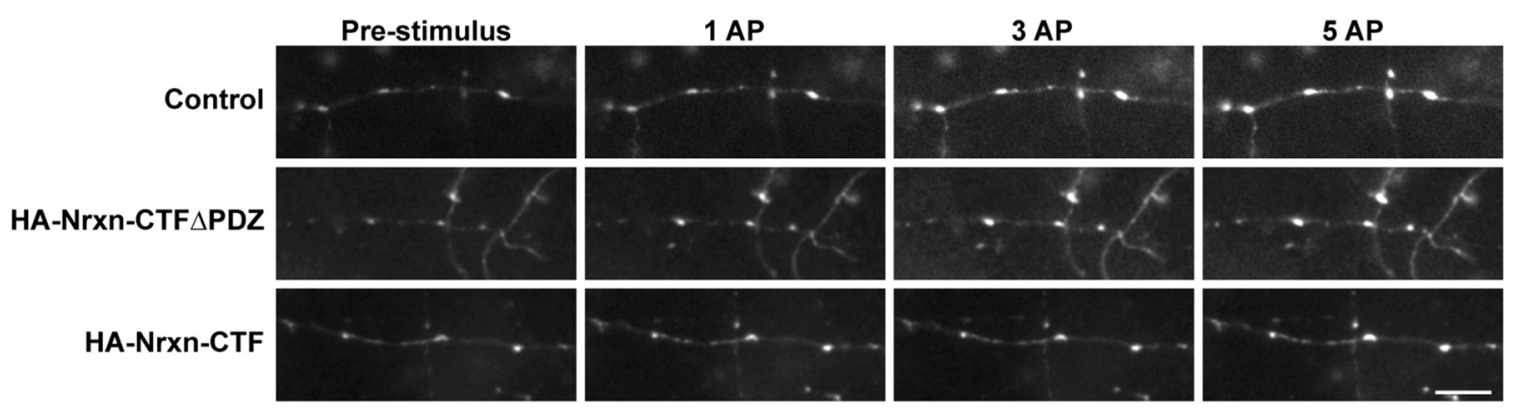

D
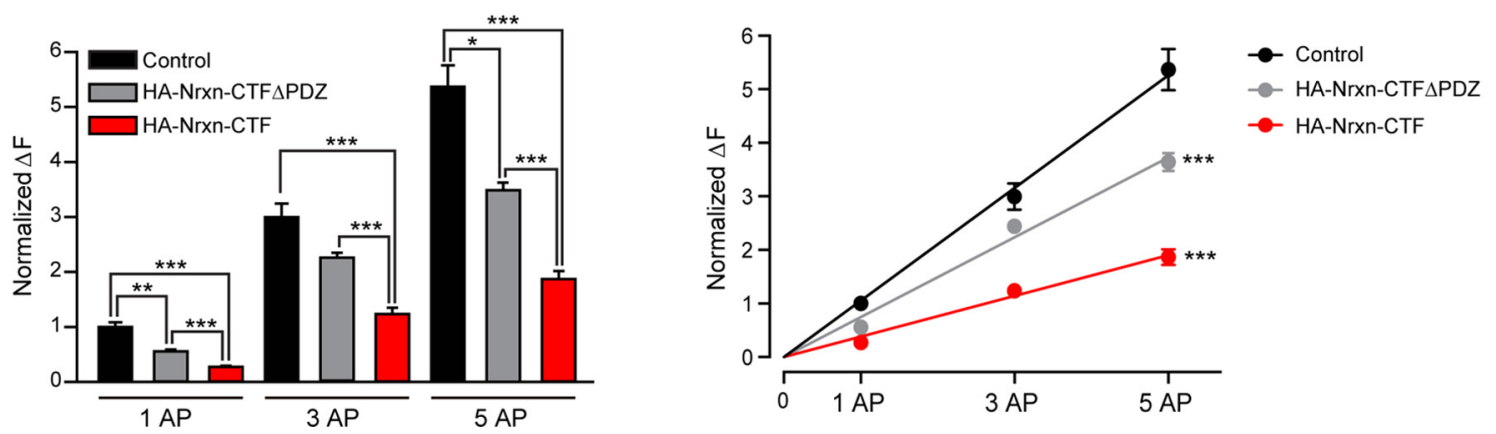

Figure 8. Impaired calcium flux at presynaptic terminals accumulating Nrxn-CTF. $A$, Cultures from PS1/fPS1; PS2 ${ }^{-/-}$hippocampal neurons were coinfected with lentivirus expressing SyGCaMP3 alone or together with lentivirus expressing $\Delta$ Cre or Cre (PS CDKO) before cotransfection of DsRed and Nlgn1. Images show peak SyGCaMP3 fluorescence of hippocampal cultures stimulated with 1, 3, and 5 APs. B, Peak amplitude ( $\Delta F$ ) of normalized SyGCaMP3 signal plotted as a bar graph (left) or as a function of the number of APs elicited (right). In each condition, quantitative analyses were performed in Nlgn1 and internal synapses not contacting Nlgn1 (internal synapses at 1 APs: control 1.0 \pm 0.080 ; control (Figure legend continues.) 
expression of HA-Nrxn-CTF strongly inhibited evoked release at 40 and $300 \mathrm{APs}$, whereas the inhibitory effect was attenuated in neurons expressing HA-Nrxn-CTF $\triangle$ PDZ despite similar localization and expression levels (normalized $\Delta F$ at $40 \mathrm{APs}$ : control $1.0 \pm 0.060$; HA-Nrxn-CTF $0.411 \pm 0.023, p<0.0001$, KruskalWallis followed by the post hoc Dunn's test; HA-Nrxn-CTF $\Delta$ PDZ $0.697 \pm 0.041, p=0.0008$, Kruskal-Wallis followed by the post hoc Dunn's test; normalized $\Delta F$ at 300 APs: control $3.580 \pm$ 0.178 ; HA-Nrxn-CTF $0.980 \pm 0.059, p<0.0001$, Kruskal-Wallis followed by the post hoc Dunn's test; HA-Nrxn-CTF $\Delta$ PDZ $1.949 \pm 0.085, p<0.0001$, Kruskal-Wallis followed by the post hoc Dunn's test; Fig. 7 B, C). CASK levels increased in axons transfected with HA-Nrxn-CTF compared with control neurons and reached intermediate levels in axons expressing HA-NrxnCTF $\triangle$ PDZ (Fig. $7 D, E$ ). These results indicated that presynaptic accumulation of Nrxn-CTF alone recapitulates the deficits in presynaptic release caused by the loss of PS activity.

\section{Nrxn-CTF mimic the inhibitory effect of PS on presynaptic calcium levels}

Synaptic vesicle release depends on the levels of calcium in the presynaptic active zone. To gain insight into the mechanism by which Nrxn-CTF impair neurotransmitter release, we measured presynaptic calcium using SyGCaMP3, a fusion of the genetically encoded calcium indicator CaMP3 to the synaptic vesicle resident protein synaptophysin (Dreosti et al., 2009; Tian et al., 2009). Calcium imaging was performed in two experimental conditions that accumulate Nrxn-CTF at presynaptic terminals, glutamatergic terminals induced by Nlgn1 in PS cDKO neurons and hippocampal neurons transfected with exogenous HA-Nrxn-CTF (Fig. 8). In each condition, presynaptic response was evoked with 1,3 , and $5 \mathrm{APs}$, a stimulus range within the linear response reported for SyGCaMP probes (Tian et al., 2009; Li et al., 2011; Akerboom et al., 2012). Stimulation of Nrxn signaling with postsynaptic Nlgn1 in control cultures locally increased evoked presynaptic calcium signal compared with untransfected synapses (Fig. 8A,B). Importantly, genetic inactivation of PS genes in PS cDKO neurons decreased evoked calcium levels at Nlgn1 synapses, leaving internal synapses contacting untransfected neurons unaffected (Fig. 8A,B). The peak SyGCaMP3 am-

\footnotetext{
$\leftarrow$

(Figure legend continued.) $\Delta$ (re 1.137 $\pm 0.061 ;$ PS CDK0 1.049 \pm 0.063 ; internal synapses at 3 APs: control $3.768 \pm 0.449$; control $\Delta$ Cre $3.438 \pm 0.215$; PS CDKO $3.299 \pm 0.220$; internal synapses at 5 APs: control $5.557 \pm 0.513$; control $\Delta$ Cre 5.129 \pm 0.264 ; PS CDK0 5.220 \pm 0.263 ; Nlgn1 synapses at 1 APs: control 1.456 \pm 0.069 ; control $\Delta C$ re 1.656 $\pm 0.010 ;$ PS CDK0 $1.238 \pm$ 0.048; Nlgn 1 synapses at 3 APs: control $5.994 \pm 0.430$; control $\Delta$ Cre $5.849 \pm 0.425$; PS cDK0 $4.142 \pm 0.201$; Nlgn 1 synapses at 5 APs: control, $8.465 \pm 0.519$; control $\Delta$ Cre $8.024 \pm 0.547$; PS CDK0 $6.283 \pm 0.300,{ }^{* *} p<0.01,{ }^{* * *} p<0.001$, Kruskal-Wallis followed by the post hoc Dunn's test). Slopes: internal control synapses $1.145 \pm 0.047$; internal $\Delta$ Cre synapses $1.060 \pm$ 0.038 ; internal PS CDK0 synapses $1.058 \pm 0.017$; Nlgn 1 synapses $1.765 \pm 0.101$, Nlgn1 $\Delta$ Cre synapses 1.695 \pm 0.106 ; Nlgn 1 PS CDK0 synapses $1.288 \pm 0.038,{ }^{* *} p<0.01,{ }^{* * *} p<0.001$, one-way ANOVA followed by the post hoc Bonferroni's test. C, Images showing peak SyGCaMP3 fluorescence of hippocampal neurons cotransfected with SyGCaMP3 alone (control) or with HA-Nrxn-CTF or HA-Nrxn-CTF $\triangle$ PDZ. D, Peak signal $(\Delta F)$ of normalized SyGCaMP3 fluorescence plotted as in $\boldsymbol{B}$ (control synapses at 1 APs $1.0 \pm 0.082$; HA-Nrxn-CTF synapses at 1 APs $0.274 \pm$ 0.017 ; HA-Nrxn-CTF $\Delta$ PDZ synapses at 1 APs $0.556 \pm 0.032$; control synapses at 3 APs $2.997 \pm$ 0.246; HA-Nrxn-CTF synapses at 3 APs $1.235 \pm 0.115$; HA-Nrxn-CTF $\Delta$ PDZ synapses at 3 APs $2.252 \pm 0.093$; control synapses at 5 APs $5.368 \pm 0.387$; HA-Nrxn-CTF synapses at 5 APs $1.869 \pm 0.148$; HA-Nrxn-CTF $\triangle$ PDZ synapses at 5 APs $3.490 \pm 0.139,{ }^{*} p<0.05,{ }^{* *} p<0.01$, ${ }^{* * *} p<0.001$, Kruskal-Wallis followed by the post hoc Dunn's test). Slopes: control $1.052 \pm$ 0.023; HA-Nrxn-CTF synapses $0.380 \pm 0.017$; HA-Nrxn-CTF $\Delta$ PDZ synapses $0.707 \pm 0.024$, ${ }^{* * *} p<0.001$ one-way ANOVA followed by the post hoc Bonferroni's test. Scale bars, $5 \mu \mathrm{m}$. Error bars indicate SEM.
}

plitude per APs, a measure of the average calcium signal evoked by a single APs, was increased at Nlgn1 synapses of control cultures compared with internal synapses $(1.145 \pm 0.047$ and $1.765 \pm 0.101$ for internal and Nlgn1 synapses of control cultures, respectively; $1.060 \pm 0.038$ and $1.695 \pm 0.106$ for internal and Nlgn1 synapses of $\Delta$ Cre cultures, respectively), whereas ablation of PS genes decreased SyGCaMP3 amplitude per APs at Nlgn1 synapses $(1.058 \pm 0.017$ and $1.288 \pm 0.038$ for internal and Nlgn1 synapses of PS cDKO synapses, respectively). Therefore, the augmented presynaptic release induced by Nlgn 1 was found to be associated with an increase in calcium levels at presynaptic terminals. Conversely, inhibition of PS function strongly decreased presynaptic calcium levels and mobilization of synaptic vesicles at Nlgn 1 synapses.

Then, we investigated whether Nrxn-CTF mediate the inhibitory response in presynaptic calcium flux induced by PS inactivation. Indeed, transfection of HA-Nrxn-CTF in hippocampal neurons inhibited evoked calcium levels at presynaptic terminals, whereas expression of HA-Nrxn-CTF $\Delta$ PDZ showed an attenuated reduction in presynaptic calcium (Fig. $8 C, D$ ). The peak SyGCaMP3 signal per AP was $1.052 \pm 0.023$ for control synapses, $0.380 \pm 0.017$ for HA-Nrxn-CTF synapses, and $0.707 \pm 0.024$ for HA-Nrxn-CTF $\Delta$ PDZ synapses. Together, these findings indicate that accumulation of Nrxn-CTF at presynaptic terminals accounts for the inhibitory effect of loss of PS function by a mechanism that reduces presynaptic calcium levels and, consequently, synaptic vesicle release.

\section{Inhibition of ectodomain shedding in Nrxn1- $\beta$ increases presynaptic release}

The presynaptic effect of overexpressed postsynaptic Nlgn 1 relies on the trans-synaptic recruitment of Nrxn- $\beta$ isoforms. In this scenario, increasing Nrxn- $\beta$ levels at the axonal surface should mimic the effect of overexpressed Nlgn1 at the postsynapse. However, it has been shown that the surface/total ratio of Nrxn- $\beta$ proteins at synapses is tightly controlled (Fu and Huang, 2010), precluding an effect of their overexpression. Our data showing that blocking ectodomain shedding increases Nrxn1- $\beta$ levels in lysates of HEK293T cells (Fig. 5) suggest the intriguing possibility that the synaptic levels of Nrxn- $\beta$ proteins are regulated by proteolysis of the extracellular domain. Interestingly, we found that expression of mutant HA-Nrxn1- $\beta \Delta 368-393$ resistant to ectodomain shedding increased Nrxn1- $\beta$ surface levels in axons of hippocampal neurons (Fig. 9). As opposed to the lack of effect of HA-Nrxn1- $\beta$, expression of HA-Nrxn1- $\beta \Delta 368-393$ proportionally increased the release of synaptic vesicles at 40 and 300 APs (Fig. 9). Therefore, increasing postsynaptic Nlgn1 levels or blocking ectodomain shedding of axonal Nrxn- $\beta$ isoforms increases synaptic levels of Nrxn- $\beta$ and stimulates presynaptic release.

\section{Discussion}

Although PS mediates the proteolytic processing of Nrxns, it has been unclear whether the synaptic clearance of Nrxn-CTF by PS is important for synaptic physiology. In this study, we performed experimental approaches based on fluorescent synaptic probes to analyze the effect of the inhibition of Nrxns processing by PS. Our results indicate that normal PS function is required to maintain neurotransmitter release and presynaptic calcium levels at glutamatergic synapses induced by Nrxn-Nlgn 1 complex. However, PS function is dispensable for the recruitment of synaptic vesicles and the induction of glutamatergic synapses mediated by Nlgn 1 . Moreover, presynaptic expression of exogenous Nrxn-CTF in 
A

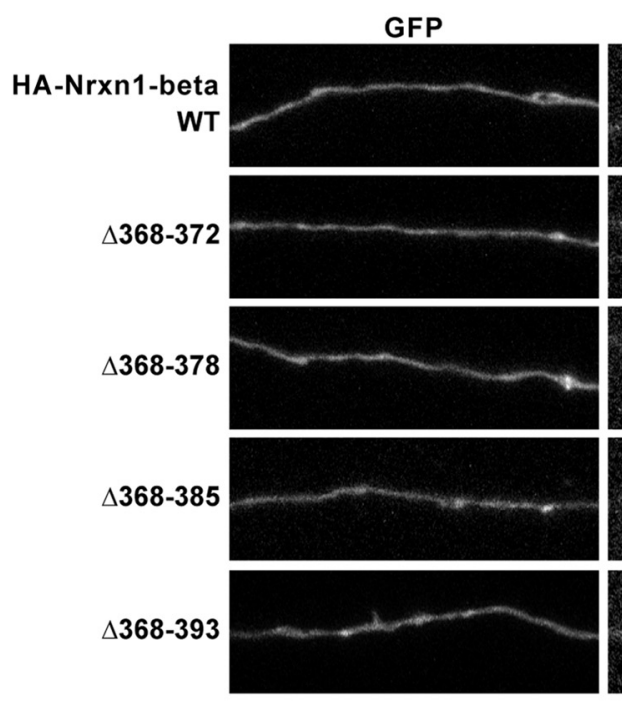

B

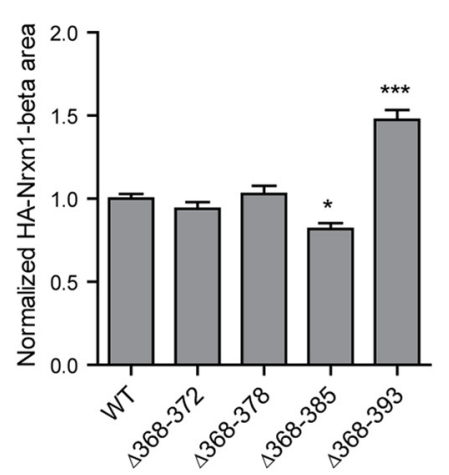

HA
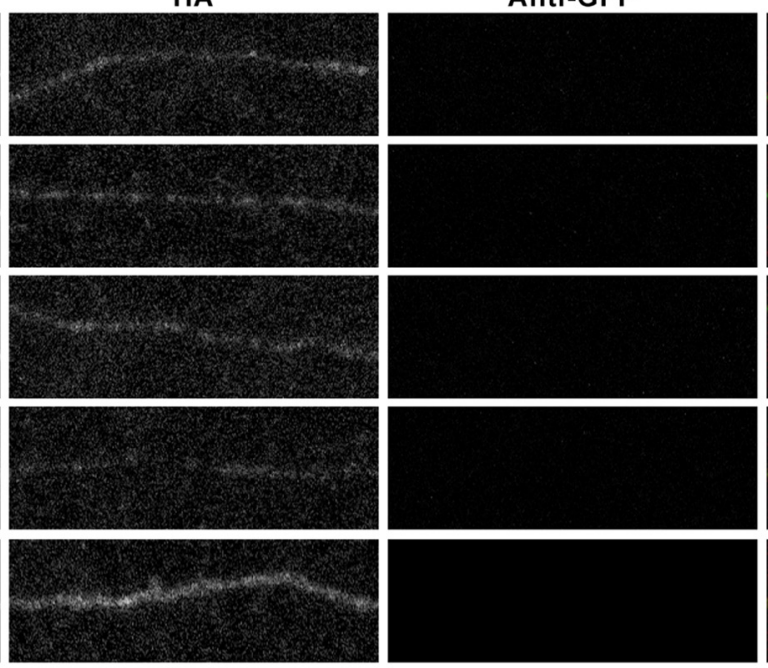

C

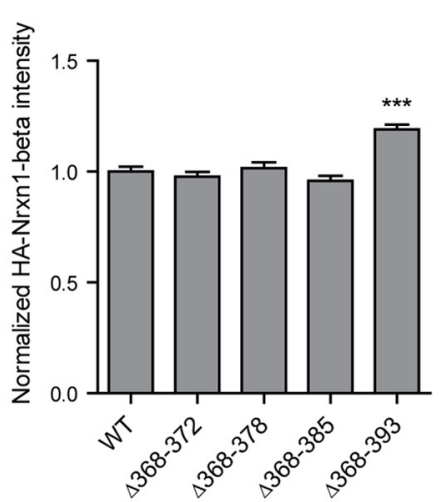

D
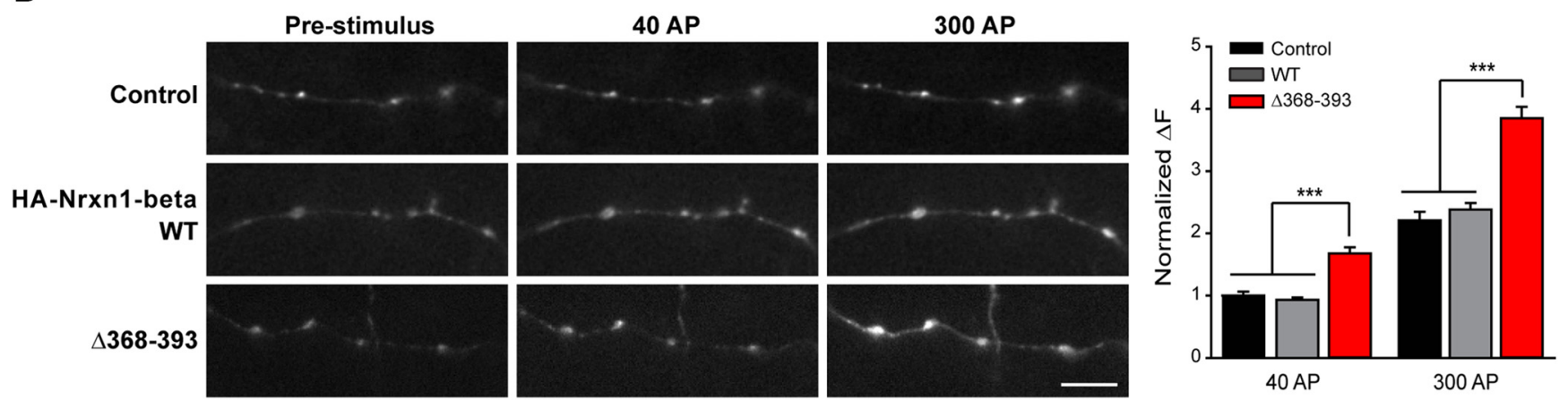

Figure 9. Effect of the inhibition of ectodomain shedding of Nrxn1- $\beta$ in axonal surface levels and presynaptic release. $A$, Immunofluorescence using HA and GFP antibodies in unpermeabilized neurons cotransfected with GFP and HA-Nrxn1- $\beta$ mutants. $\boldsymbol{B}$, Normalized HA-Nrxn1- $\beta$ area in transfected axons (wild-type $1.0 \pm 0.028 ; \Delta 368-3720.939 \pm 0.040 ; \Delta 368-3781.027 \pm 0.050$; $\Delta 368-3850.817 \pm 0.036 ; \Delta 368-3931.474 \pm 0.058,{ }^{*} p<0.05,{ }^{* * *} p<0.001$, one-way ANOVA followed by the post hoc Bonferroni's test). C, Normalized HA-Nrxn1- $\beta$ intensity in transfected axons (wild-type $1.0 \pm 0.022 ; \Delta 368-3720.977 \pm 0.021 ; \Delta 368-3781.015 \pm 0.027 ; \Delta 368-3850.959 \pm 0.023 ; \Delta 368-3931.190 \pm 0.022$, ${ }^{* * *} p<0.001$, Kruskal-Wallis with Dunn's multiple-comparisons test). Data are shown from 15-18 images from two independent experiments from different cultures. $D$, Images showing Synaptophysin-pHluorin (SypHy) fluorescence and quantifications of hippocampal cultures expressing SypHy alone (control) or together with wild-type HA-Nrxn1- $\beta$ or mutant HA-Nrxn1- $\beta \Delta 368-393$ (control synapses at 40 APs $1.0 \pm 0.064$; HA-Nrxn1- $\beta$ synapses at 40 APs $0.932 \pm 0.039$; HA-Nrxn1- $\beta \Delta 368-393$ synapses at 40 APs $1.677 \pm 1.100$; control synapses at 300 APs $2.207 \pm 0.137$; HA-Nrxn1- $\beta$ synapses at 300 APs $2.383 \pm$ 0.102 ; HA-Nrxn1- $\beta \Delta 368-393$ synapses at 300 APs $3.850 \pm 0.182,{ }^{* * *} p<0.001$, Kruskal-Wallis followed by the post hoc Dunn's test). Scale bar, $5 \mu \mathrm{m}$. Error bars indicate SEM.

hippocampal neurons decreases synaptic vesicle release and presynaptic calcium levels, thereby recapitulating the effect caused by inactivation of PS activity. The inhibitory effect of Nrxn-CTF is attenuated by the deletion of the last three C-terminal residues, which form a functional PDZ-binding domain in Nrxn-CTF. Together, our data indicate that accumulation of Nrxn-CTF due to impaired PS activity is deleterious to the function of glutamatergic synapses.

Due to the role of Nrxns in presynaptic function, the accumulation of Nrxn-CTF at glutamatergic terminals favored a potential impact of Nrxn processing in the synaptic phenotype associated with the loss of PS function. However, the challenges in assigning synaptic 
phenotypes to specific PS substrates and the complexity in analyzing presynaptic function have precluded answering these questions. In this study, we analyzed the functional implications of the proteolytic processing of Nrxns at glutamatergic terminals. Electrophysiological recording of Nlgn1-expressing neurons supported the notion of a trans-synaptic mechanism that increases release probability at glutamatergic synapses (Futai et al., 2007). Surprisingly, expression of Nrxn1- $\beta$ failed to increase neurotransmitter release, whereas expression of a Nrxn1- $\beta$ deletion mutant lacking the cytoplasmic tail decreases neurotransmitter release while preserving the trans-synaptic interaction with Nlgn1 (Futai et al., 2007; Wittenmayer et al., 2009; Rabaneda et al., 2014). These data suggested that the function of Nrxn- $\beta$ isoforms at glutamatergic terminals is tightly regulated by postsynaptic binding partners. Using synaptic probes to visualize presynaptic release directly, we found that Nlgn 1 enhances the size of the different pools of synaptic vesicles at single terminals. Moreover, calcium-imaging experiments showed that expression of Nlgn1 increases APs-evoked calcium levels at contacting terminals. Because the probability of neurotransmitter release correlates with the size of the RRP (Murthy and Stevens, 1999), our data are consistent with a role of Nlgn 1 as a rate-limiting factor that regulates the number of functional release sites at contacting terminals by stimulating trans-synaptic signaling through Nrxn- $\beta$ isoforms. In this regard, blocking ectodomain shedding in HA-Nrxn 1- $\beta$ increases presynaptic release and the levels of the protein at the axonal membrane. Therefore, the increase in presynaptic release sites mediated by Nrxn- $\beta$ can at least partly explain the positive effect of Nlgn1 in neurotransmitter release probability.

Importantly, we found that ablation of PS genes in PS cDKO neurons or inactivation of PS activity with genetic and chemical approaches reduced the activity-dependent mobilization of synaptic vesicles and decreased calcium levels at Nlgn1 synapses. Although Nrxn-Nlgn1 signaling increases the number of glutamatergic synapses (Dean et al., 2003; Boucard et al., 2005; Chih et al., 2006), the increase in density and intensity of synaptic vesicle markers induced by Nlgn 1 was not altered in neurons lacking PS activity, suggesting a selective role of PS in the release of synaptic vesicles downstream of Nrxn activation. The most plausible explanation for these results is the generation of a PS substrate at Nlgn1-induced presynaptic terminals, the local accumulation of which renders Nlgn1 synapses susceptible to the loss of PS function. It is noteworthy to mention that, whereas Nrxn-CTF are generated in neurons with impaired PS activity (Saura et al., 2011; this study), presynaptic release in synapses not contacting Nlgn1 was only slightly reduced at 300 APs by the loss of PS activity. Importantly, the immunoreactivity for a cyto-Nrxn antibody was found increased at Nlgn 1 synapses of PS cDKO cultures, expanding our previous data using PS1 dominant-negative mutants (Saura et al., 2011). Our data favor two potential nonexcluding mechanisms for the local generation of Nrxn-CTF at glutamatergic terminals: (1) increase Nrxn availability through adhesion to postsynaptic Nlgn1 or (2) initiation of the proteolytic processing by an activity-dependent mechanism mediated by NMDA/ AMPA receptor signaling. It has been reported that Nlng1 increases the recruitment of NMDA receptors (Budreck et al., 2013), which in turn can become activated by glutamate released from the apposing terminal. Therefore, the higher susceptibility of Nrxn-Nlgn1 synapses to the loss of PS function correlates with increased concentration of Nrxn-CTF at presynaptic terminals.

The accumulation of Nrxn-CTF is governed by the balance of the cleavage at the extracellular domain and the clearance of the resulting Nrxn-CTF by PS. Using deletion mutants in the extracellular domain of human Nrxn $1-\beta$, we identified a short region in the juxtamembrane domain comprising residues 386-393 responsible for the release of the ectodomain and the generation of Nrxn-CTF. The predicted Nrxn-CTF contain the transmembrane and cytoplasmic domains of Nrxns and this is conserved among Nrxn isoforms. Moreover, we demonstrated that inhibition of PS activity in heterologous and neuronal cells increases the levels of an HA-tagged Nrxn-CTF construct containing the last 85 residues of human Nrxn1, further indicating that Nrxn1 C-terminal fragments starting at V388 function as a PS substrate, as shown recently for Nrxn3 (Borcel et al., 2016). One important consequence of the identification of the cleavage site that generates Nrxn-CTF is that it enabled the functional study of the proteolytic processing of Nrxns by PS without broadly affecting PS activity toward other neuronal substrates. A key finding of our study is that presynaptic accumulation of exogenous Nrxn-CTF strongly decreases evoked calcium influx at axon terminals and consequently synaptic vesicle release, thereby mimicking the inhibitory effect of the loss of PS function. The inhibition in presynaptic function induced by Nrxn-CTF is mediated by a synaptic mechanism that depends on the integrity of the cytoplasmic tail, as shown by the attenuated effect of a deletion mutant that lacks the PDZ-binding domain of Nrxn-CTF. The increased CASK levels indicate that accumulation of ligands that interact with Nrxn-CTF might interfere with presynaptic signaling occurring at contact sites downstream of the cytoplasmic tail of Nrxns, thereby uncoupling presynaptic release from trans-synaptic adhesion. Regions other than the PDZ-binding domain could contribute to the intermediate CASK levels accumulated by the Nrxn-CTF $\Delta$ PDZ mutant. The cytoplasmic tail of Nrxn1 contains a conserved juxtamembrane domain that binds indirectly to CASK through neuronal Protein 4.1N (Biederer and Südhof, 2001). Whether CASK levels are increased by this or by an alternative mechanism remains to be investigated, however, it is interesting that the inhibition in presynaptic release caused by Nrxn-CTF and Nrxn-CTF $\Delta$ PDZ constructs correlates with the levels of accumulated CASK.

Collectively, our data indicate that stimulation of Nrxn-Nlgn1 trans-synaptic signaling increases the number of functional release sites at contacting presynaptic terminals. Synaptic activity initiates the proteolytic shedding of the extracellular domain of Nrxns by a NMDA/AMPA receptor-dependent signaling, which results in the generation of Nrxn-CTF. Interestingly, Nrxn1- $\beta$ ectodomain accelerates the activity-dependent shedding of Nlgn1, which in turn can modulate the retrograde pathway that controls presynaptic release probability (Peixoto et al., 2012; Suzuki et al., 2012). Therefore, the Nrxn-Nlgn 1 signaling pathway is limited by the coordinated activity-dependent processing of their members. Nrxn-CTF produced at glutamatergic terminals are further processed by PS/gamma-secretase complex, leading to its synaptic clearance. Loss of PS activity with chemical inhibitors or by genetic inactivation accumulates Nrxn-CTF at presynaptic terminals, which reduces evoked presynaptic calcium flux selectively and, as a consequence, exocytosis of synaptic vesicles. Therefore, accumulation of Nrxn-CTF explains the inhibitory effects caused by loss of PS function at glutamatergic synapses. It has been proposed that FAD-linked PS mutations produce a partial loss-offunction mechanism, reducing the proteolytic activity of PS/ gamma-secretase complex toward its substrates (Shen and Kelleher, 2007; Sun et al., 2017), including Nrxns (Bot et al., 2011; Saura et al., 2011). Importantly, genetic inactivation of PS genes in excitatory neurons results in deficits in glutamatergic release 
by a presynaptic mechanism, followed by age-dependent neurodegeneration (Saura et al., 2004; Zhang et al., 2009, 2010). Remarkably, the accumulation of Nrxn-CTF at glutamatergic terminals of PS cDKO mice occurs at an early developmental time that coincides with deficits in glutamatergic transmission, but precedes neurodegeneration (Saura et al., 2011). Whereas the consequences of impaired PS activity toward specific substrates remain to be clarified fully, our data suggest that presynaptic accumulation of NrxnCTF can contribute to the synaptic and neuronal deficits associated with the loss of PS function in FAD.

\section{References}

Akerboom J, et al. (2012) Optimization of a GCaMP calcium indicator for neural activity imaging. J Neurosci 32:13819-13840. CrossRef Medline

Aoto J, Földy C, Ilcus SM, Tabuchi K, Südhof TC (2015) Distinct circuitdependent functions of presynaptic neurexin-3 at GABAergic and glutamatergic synapses. Nat Neurosci 18:997-1007. CrossRef Medline

Baudouin S, Scheiffele P (2010) SnapShot: neuroligin-neurexin complexes. Cell 141:908, 908.e901. CrossRef Medline

Bie B, Wu J, Yang H, Xu JJ, Brown DL, Naguib M (2014) Epigenetic suppression of neuroligin 1 underlies amyloid-induced memory deficiency. Nat Neurosci 17:223-231. CrossRef Medline

Biederer T, Südhof TC (2001) CASK and protein 4.1 support F-actin nucleation on neurexins. J Biol Chem 276:47869-47876. CrossRef Medline

Borcel E, Palczynska M, Krzisch M, Dimitrov M, Ulrich G, Toni N, Fraering PC (2016) Shedding of neurexin $3 \beta$ ectodomain by ADAM10 releases a soluble fragment that affects the development of newborn neurons. Sci Rep 6:39310. CrossRef Medline

Bot N, Schweizer C, Ben Halima S, Fraering PC (2011) Processing of the synaptic cell-adhesion molecule neurexin-3\{beta\} by Alzheimer's disease \{alpha\}- and \{gamma\}-secretases. J Biol Chem 286:2762-2773. CrossRef Medline

Boucard AA, Chubykin AA, Comoletti D, Taylor P, Südhof TC (2005) A splice code for trans-synaptic cell adhesion mediated by binding of neuroligin 1 to $\alpha$ - and $\beta$-neurexins. Neuron 48:229-236. CrossRef Medline

Budreck EC, Kwon OB, Jung JH, Baudouin S, Thommen A, Kim HS, Fukazawa Y, Harada H, Tabuchi K, Shigemoto R, Scheiffele P, Kim JH (2013) neuroligin-1 controls synaptic abundance of NMDA-type glutamate receptors through extracellular coupling. Proc Natl Acad Sci U S A 110: 725-730. CrossRef Medline

Butz S, Okamoto M, Südhof TC (1998) A tripartite protein complex with the potential to couple synaptic vesicle exocytosis to cell adhesion in brain. Cell 94:773-782. CrossRef Medline

Camacho-Garcia RJ, Planelles MI, Margalef M, Pecero ML, Martínez-Leal R, Aguilera F, Vilella E, Martinez-Mir A, Scholl FG (2012) Mutations affecting synaptic levels of neurexin- $1 \beta$ in autism and mental retardation. Neurobiol Dis 47:135-143. CrossRef Medline

Camacho-Garcia RJ, Hervás A, Toma C, Balmaña N, Cormand B, MartinezMir A, Scholl FG (2013) Rare variants analysis of neurexin- $1 \beta$ in autism reveals a novel start codon mutation affecting protein levels at synapses. Psychiatr Genet 23:262-266. CrossRef Medline

Chih B, Gollan L, Scheiffele P (2006) Alternative splicing controls selective trans-synaptic interactions of the neuroligin-neurexin complex. Neuron 51:171-178. CrossRef Medline

Dean C, Scholl FG, Choih J, DeMaria S, Berger J, Isacoff E, Scheiffele P (2003) neurexin mediates the assembly of presynaptic terminals. Nat Neurosci 6:708-716. CrossRef Medline

Dreosti E, Odermatt B, Dorostkar MM, Lagnado L (2009) A genetically encoded reporter of synaptic activity in vivo. Nat Methods 6:883-889. CrossRef Medline

Edelstein A, Amodaj N, Hoover K, Vale R, Stuurman N (2010) Computer control of microscopes using microManager. Curr Protoc Mol Biol Chapter 14:Unit14.20. CrossRef Medline

Fu Y, Huang ZJ (2010) Differential dynamics and activity-dependent regulation of $\alpha$ - and $\beta$-neurexins at developing GABAergic synapses. Proc Natl Acad Sci U S A 107:22699-22704. CrossRef Medline

Fuccillo MV, Földy C, Gökce Ö, Rothwell PE, Sun GL, Malenka RC, Südhof TC (2015) Single-cell mRNA profiling reveals cell-type-specific expression of neurexin isoforms. Neuron 87:326-340. CrossRef Medline

Futai K, Kim MJ, Hashikawa T, Scheiffele P, Sheng M, Hayashi Y (2007) Retrograde modulation of presynaptic release probability through signal- ing mediated by PSD-95-neuroligin. Nat Neurosci 10:186-195. CrossRef Medline

Gascón S, Paez-Gomez JA, Díaz-Guerra M, Scheiffele P, Scholl FG (2008) Dual-promoter lentiviral vectors for constitutive and regulated gene expression in neurons. J Neurosci Methods 168:104-112. CrossRef Medline

Granseth B, Odermatt B, Royle SJ, Lagnado L (2006) Clathrin-mediated endocytosis is the dominant mechanism of vesicle retrieval at hippocampal synapses. Neuron 51:773-786. CrossRef Medline

Grootjans JJ, Reekmans G, Ceulemans H, David G (2000) Syntenin-syndecan binding requires syndecan-synteny and the co-operation of both PDZ domains of syntenin. J Biol Chem 275:19933-19941. CrossRef Medline

Hass MR, Sato C, Kopan R, Zhao G (2009) Presenilin: RIP and beyond. Semin Cell Dev Biol 20:201-210. CrossRef Medline

Ho A, Morishita W, Atasoy D, Liu X, Tabuchi K, Hammer RE, Malenka RC, Südhof TC (2006) Genetic analysis of Mint/X11 proteins: essential presynaptic functions of a neuronal adaptor protein family. J Neurosci 26: 13089-13101. CrossRef Medline

Huntley GW (2012) Synaptic circuit remodelling by matrix metalloproteinases in health and disease. Nat Rev Neurosci 13:743-757. CrossRef Medline

Ichtchenko K, Hata Y, Nguyen T, Ullrich B, Missler M, Moomaw C, Südhof TC (1995) neuroligin 1: a splice site-specific ligand for $\beta$-neurexins. Cell 81:435-443. CrossRef Medline

Jamain S, Quach H, Betancur C, Råstam M, Colineaux C, Gillberg IC, Soderstrom H, Giros B, Leboyer M, Gillberg C, Bourgeron T, Paris Autism Research International Sibpair S (2003) Mutations of the X-linked genes encoding neuroligins NLGN3 and NLGN4 are associated with autism. Nat Genet 34:27-29. CrossRef Medline

Kim HG, et al. (2008) Disruption of neurexin 1 associated with autism spectrum disorder. Am J Hum Genet 82:199-207. CrossRef Medline

Ko J, Fuccillo MV, Malenka RC, Südhof TC (2009) LRRTM2 functions as a neurexin ligand in promoting excitatory synapse formation. Neuron 64: 791-798. CrossRef Medline

Laumonnier F, Bonnet-Brilhault F, Gomot M, Blanc R, David A, Moizard MP, Raynaud M, Ronce N, Lemonnier E, Calvas P, Laudier B, Chelly J, Fryns JP, Ropers HH, Hamel BC, Andres C, Barthélémy C, Moraine C, Briault S (2004) X-linked mental retardation and autism are associated with a mutation in the NLGN4 gene, a member of the neuroligin family. Am J Hum Genet 74:552-557. CrossRef Medline

Li H, Foss SM, Dobryy YL, Park CK, Hires SA, Shaner NC, Tsien RY, Osborne LC, Voglmaier SM (2011) Concurrent imaging of synaptic vesicle recycling and calcium dynamics. Front Mol Neurosci 4:34. CrossRef Medline

Lowther C, et al. (2017) Molecular characterization of NRXN1 deletions from 19,263 clinical microarray cases identifies exons important for neurodevelopmental disease expression. Genet Med 19:53-61. CrossRef Medline

Martinez-Mir A, González-Pérez A, Gayán J, Antúnez C, Marín J, Boada M, Lopez-Arrieta JM, Fernández E, Ramírez-Lorca R, Sáez ME, Ruiz A, Scholl FG, Real LM (2013) Genetic study of neurexin and neuroligin genes in Alzheimer's disease. J Alzheimers Dis 35:403-412. CrossRef Medline

Murthy VN, Stevens CF (1999) Reversal of synaptic vesicle docking at central synapses. Nat Neurosci 2:503-507. CrossRef Medline

Murthy VN, Schikorski T, Stevens CF, Zhu Y (2001) Inactivity produces increases in neurotransmitter release and synapse size. Neuron 32:673682. CrossRef Medline

Nagy V, Bozdagi O, Matynia A, Balcerzyk M, Okulski P, Dzwonek J, Costa RM, Silva AJ, Kaczmarek L, Huntley GW (2006) Matrix metalloproteinase-9 is required for hippocampal late-phase long-term potentiation and memory. J Neurosci 26:1923-1934. CrossRef Medline

Peixoto RT, Kunz PA, Kwon H, Mabb AM, Sabatini BL, Philpot BD, Ehlers MD (2012) Transsynaptic signaling by activity-dependent cleavage of neuroligin-1. Neuron 76:396-409. CrossRef Medline

Prox J, Bernreuther C, Altmeppen H, Grendel J, Glatzel M, D'Hooge R, Stroobants S, Ahmed T, Balschun D, Willem M, Lammich S, Isbrandt D, Schweizer M, Horré K, De Strooper B, Saftig P (2013) Postnatal disruption of the disintegrin/metalloproteinase ADAM10 in brain causes epileptic seizures, learning deficits, altered spine morphology, and defective synaptic functions. J Neurosci 33:12915-12928, 12928a. CrossRef Medline

Rabaneda LG, Robles-Lanuza E, Nieto-González JL, Scholl FG (2014) neurexin dysfunction in adult neurons results in autistic-like behavior in mice. Cell Rep 8:338-346. CrossRef Medline 
Rujescu D, et al. (2009) Disruption of the neurexin 1 gene is associated with schizophrenia. Hum Mol Genet 18:988-996. CrossRef Medline

Saura CA, Choi SY, Beglopoulos V, Malkani S, Zhang D, Shankaranarayana Rao BS, Chattarji S, Kelleher RJ 3rd, Kandel ER, Duff K, Kirkwood A, Shen J (2004) Loss of presenilin function causes impairments of memory and synaptic plasticity followed by age-dependent neurodegeneration. Neuron 42:23-36. CrossRef Medline

Saura CA, Servián-Morilla E, Scholl FG (2011) presenilin/ $\gamma$-secretase regulates neurexin processing at synapses. PLoS One 6:e19430. CrossRef Medline

Scheiffele P, Fan J, Choih J, Fetter R, Serafini T (2000) neuroligin expressed in nonneuronal cells triggers presynaptic development in contacting axons. Cell 101:657-669. CrossRef Medline

Schikorski T, Stevens CF (2001) Morphological correlates of functionally defined synaptic vesicle populations. Nat Neurosci 4:391-395. CrossRef Medline

Schreiner D, Nguyen TM, Russo G, Heber S, Patrignani A, Ahrné E, Scheiffele $P$ (2014) Targeted combinatorial alternative splicing generates brain region-specific repertoires of neurexins. Neuron 84:386-398. CrossRef Medline

Schreiner D, Simicevic J, Ahrné E, Schmidt A, Scheiffele P (2015) Quantitative isoform-profiling of highly diversified recognition molecules. Elife 4:e07794. Medline

Shen J, Kelleher RJ 3rd (2007) The presenilin hypothesis of Alzheimer's disease: evidence for a loss-of-function pathogenic mechanism. Proc Natl Acad Sci U S A 104:403-409. CrossRef Medline

Sindi IA, Tannenberg RK, Dodd PR (2014) Role for the neurexin-neuroligin complex in Alzheimer's disease. Neurobiol Aging 35:746-756. CrossRef Medline

Sun L, Zhou R, Yang G, Shi Y (2017) Analysis of 138 pathogenic mutations in presenilin- 1 on the in vitro production of $\mathrm{A} \beta 42$ and $\mathrm{A} \beta 40$ peptides by $\gamma$-secretase. Proc Natl Acad Sci U S A 114:E476-E485. CrossRef Medline

Suzuki K, Hayashi Y, Nakahara S, Kumazaki H, Prox J, Horiuchi K, Zeng M, Tanimura S, Nishiyama Y, Osawa S, Sehara-Fujisawa A, Saftig P, Yokoshima S, Fukuyama T, Matsuki N, Koyama R, Tomita T, Iwatsubo T (2012) Activity-dependent proteolytic cleavage of neuroligin-1. Neuron 76:410-422. CrossRef Medline
Tabuchi K, Südhof TC (2002) Structure and evolution of neurexin genes: insight into the mechanism of alternative splicing. Genomics 79:849859. CrossRef Medline

Taniguchi H, Gollan L, Scholl FG, Mahadomrongkul V, Dobler E, Limthong N, Peck M, Aoki C, Scheiffele P (2007) Silencing of neuroligin function by postsynaptic neurexins. J Neurosci 27:2815-2824. CrossRef Medline

Tian L, Hires SA, Mao T, Huber D, Chiappe ME, Chalasani SH, Petreanu L, Akerboom J, McKinney SA, Schreiter ER, Bargmann CI, Jayaraman V, Svoboda K, Looger LL (2009) Imaging neural activity in worms, flies and mice with improved GCaMP calcium indicators. Nat Methods 6:875881. CrossRef Medline

Tristán-Clavijo E, Camacho-Garcia RJ, Robles-Lanuza E, Ruiz A, van der Zee J, Van Broeckhoven C, Hernandez I, Martinez-Mir A, Scholl FG (2015) A truncating mutation in Alzheimer's disease inactivates neuroligin-1 synaptic function. Neurobiol Aging 36:3171-3175. CrossRef Medline

Tu H, Nelson O, Bezprozvanny A, Wang Z, Lee SF, Hao YH, Serneels L, De Strooper B, Yu G, Bezprozvanny I (2006) presenilins form ER Ca2+ leak channels, a function disrupted by familial Alzheimer's disease-linked mutations. Cell 126:981-993. CrossRef Medline

Watanabe H, Smith MJ, Heilig E, Beglopoulos V, Kelleher RJ 3rd, Shen J (2009) Indirect regulation of presenilins in CREB-mediated transcription. J Biol Chem 284:13705-13713. CrossRef Medline

Wittenmayer N, Körber C, Liu H, Kremer T, Varoqueaux F, Chapman ER, Brose N, Kuner T, Dresbach T (2009) Postsynaptic neuroligin1 regulates presynaptic maturation. Proc Natl Acad Sci U S A 106:13564-13569. CrossRef Medline

Xia D, Watanabe H, Wu B, Lee SH, Li Y, Tsvetkov E, Bolshakov VY, Shen J, Kelleher RJ 3rd (2015) presenilin-1 knockin mice reveal loss-of-function mechanism for familial Alzheimer's disease. Neuron 85:967-981. CrossRef Medline

Zhang C, Wu B, Beglopoulos V, Wines-Samuelson M, Zhang D, Dragatsis I, Südhof TC, Shen J (2009) presenilins are essential for regulating neurotransmitter release. Nature 460:632-636. CrossRef Medline

Zhang D, Zhang C, Ho A, Kirkwood A, Südhof TC, Shen J (2010) Inactivation of presenilins causes pre-synaptic impairment prior to postsynaptic dysfunction. J Neurochem 115:1215-1221. CrossRef Medline 